\title{
Chern-Simons matter theories and higher spin gravity
}

\section{Ergin Sezgin, ${ }^{a}$ Evgeny D. Skvortsov ${ }^{b, c}$ and Yaodong Zhu ${ }^{a}$}

${ }^{a}$ Mitchell Institute for Fundamental Physics and Astronomy, Texas A\&M University, College Station, TX r7843, U.S.A.

${ }^{b}$ Arnold Sommerfeld Center for Theoretical Physics, Ludwig-Maximilians University Munich, Theresienstr. 37, D-80333 Munich, Germany

${ }^{c}$ Theory Department, Lebedev Institute of Physics, Leninsky ave. 53, 119991 Moscow, Russia

E-mail: sezgin@physics.tamu.edu, skvortsov@lpi.ru, yaodongmatt@physics.tamu.edu

ABSTRACT: We compute the parity violating three point amplitudes with one scalar leg in higher spin gravity and compare results with those of Chern-Simons matter theories. The three-point correlators of the free boson, free fermion, critical vector model and Gross-Neveu model are reproduced including the dependence on the Chern-Simons coupling. We also perform a simple test of the modified higher spin equations proposed in arXiv: 1605.02662 and find that the results are consistent with the AdS/CFT correspondence.

Keywords: AdS-CFT Correspondence, Higher Spin Symmetry, Conformal Field Theory, $1 / \mathrm{N}$ Expansion

ArXiv EPrint: 1705.03197 


\section{Contents}

1 Introduction $\quad 2$

2 CFT $\quad 6$

2.1 General structure of the correlators 6

2.2 Free boson 8

2.3 Free fermion 9

$\begin{array}{llr}2.4 & \text { Critical boson } & 10\end{array}$

2.5 Critical fermion 11

2.6 Chern-Simons matter theories 11

3 Higher spin interactions $\quad 12$

4 Kinematics of the boundary-to-bulk propagators $\quad 14$

$\begin{array}{lll}4.1 & \text { Definitions } & 15\end{array}$

$\begin{array}{lll}4.2 & \text { Algebraic identities } & 16\end{array}$

$\begin{array}{lll}4.3 & \text { Inversion map } & 16\end{array}$

5 Vertices and propagators $\quad 16$

$\begin{array}{lll}5.1 \text { Propagators } & 17\end{array}$

$\begin{array}{lll}5.2 \text { Vertices } & 17\end{array}$

6 Computation of the cubic amplitude $\quad 19$

6.1 Leading coefficients 21

6.2 Complete dictionary 23

6.3 Complete three-point functions 23

6.3.1 Type-A, free boson 24

6.3.2 Type-B, free fermion 25

$\begin{array}{ll}\text { 6.3.3 Type-A, critical boson } & 26\end{array}$

6.3.4 Type-B, critical fermion 26

$\begin{array}{lll}\text { 6.3.5 Summary } & 27\end{array}$

$\begin{array}{lll}7 & \text { Discussion } & 27\end{array}$

$\begin{array}{lr}\text { A Notation and conventions } & 29\end{array}$

B Triple sums $\quad 30$

B.1 Type-A, free boson 30

B.2 Type-B, free fermion 31

B.3 Type-A, critical boson 32

B.4 Type-B, critical fermion 33 


\section{Introduction}

Higher spin (HS) theories provide simple models of AdS/CFT duality where the dual conformal field theories have matter in vector representation of the gauge group [1-4]. Such CFTs have a rather moderate growth of states and are usually exactly solvable in the large- $N$ limit. Moreover, free CFTs fall into this class too and are, in fact, generic duals of HS theories. Boundary values of HS gauge fields are sources of conserved tensors whose presence is a distinguished feature of free CFTs in $d \geq 3$ [5-8]. They also occur in the strict $N=\infty$ limit of interacting vector models. In this paper we study a rich class of $A d S_{4} / C F T_{3}$ dualities between Chern-Simons matter theories [9, 10] and four-dimensional HS theories [9]. Free theories are usually isolated as well as vector models, but it turns out that in $3 d$ there is a one-parameter family of theories that are defined by coupling free or interacting vector models to Chern-Simons gauge field, which does not introduce any new local operators and does not break conformal symmetry. Parity is broken in Chern-Simons matter theories and correlation functions of HS currents have a very special structure [11]: they consist of several terms, some of which occur in free theories and some other break parity. In [12] these parity-breaking terms were found by direct computation in ChernSimons matter theories. In this paper we reproduce some of these structure from the $A d S_{4}$ side by computing the three-point amplitudes involving a scalar field. The subsector of HS theory needed for the computation of this amplitude is distinguished by the fact that the interactions are local and are fixed by the HS algebra structure constants and does not require dealing with non-localities present in the Vasiliev equations.

The emerging relations between various CFTs in three dimensions and HS theories in four dimensions are depicted in the diagram; see [13] for a review. In the upper left corner is free bosonic CFT, which RG flows to critical boson model under $\left(\phi^{2}\right)^{2}$ deformation. On the upper right is the critical fermion (Gross-Neveu) model with $(\bar{\psi} \psi)^{2}$ interactions, which RG flows to the free fermion CFT. The right arrow at the top denotes the coupling of level $k$ Chern-Simons theory and dialing of the 't Hoof coupling $\lambda_{b}=N / k,{ }^{1}$ while the left arrow at the top denotes the coupling of CS theory to the critical fermion model and dialing up the associated 't Hooft coupling $\lambda_{f}$. Similarly, the left arrow at the bottom denotes the coupling of CS theory to the free fermion model and dialing up the 't Hoof coupling $\lambda_{f}$, and the right arrow at the bottom denotes the coupling of CS theory to critical boson model and dialing up the 't Hooft coupling $\lambda_{b}$. The dialings of the effective 't Hooft couplings $\tilde{\lambda}$ are always from 0 to $\infty$.

It turns out that the spectra of singlet operators coincide in free fermion/critical boson and free boson/critical fermion pairs in the $N=\infty$ limit. This observation together with many other tests has led to the conjecture of three-dimensional bosonization $[9,11,14,16]$ : the bosonic theory at any $\lambda_{b}$ and $N_{b}$ can be equivalently described as the dual fermionic theory at some other $\lambda_{f}$ and $N_{f}$. More general dualities can be found in [16-18]. The theory that interpolates between the free fermion and critical boson models is called quasifermionic (or CS-fermion) model. The theory that interpolates between free boson and

\footnotetext{
${ }^{1}$ In practice, it is convenient to pass to effective $\tilde{N}$ and $\tilde{\lambda}$, which we will use throughout the paper. The relation to the microscopical parameters $N$ and $\lambda$ requires computation of some correlators, see [14, 15].
} 


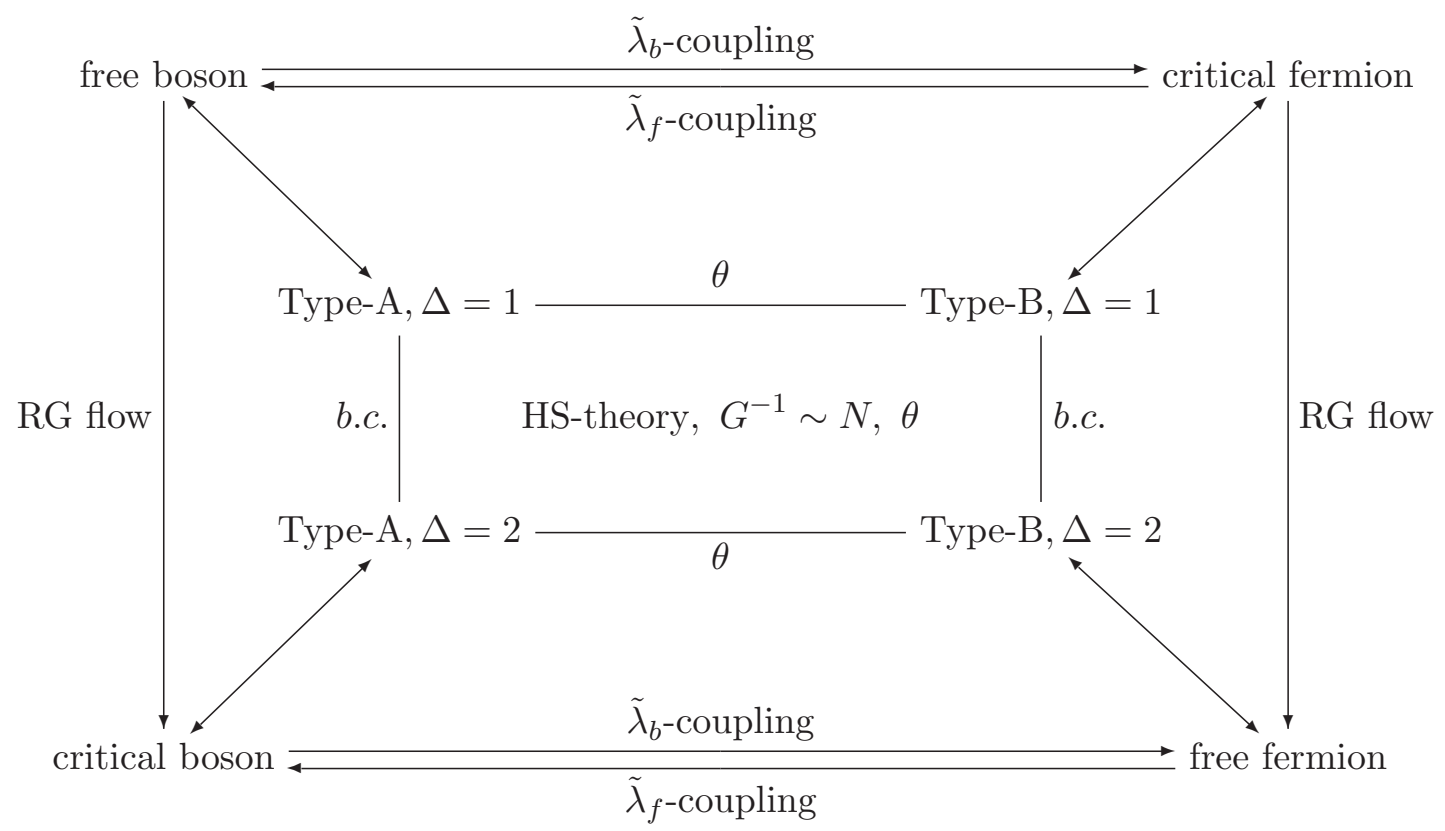

Figure 1. Schematic depiction of the relationships between various CFTs in three dimensions and higher spin gravity in four dimensions. The latter has two coupling constants, namely $(G, \theta)$, and $\Delta=1, \Delta=2$ or mixed boundary conditions can be imposed on the scalar field.

critical fermion models is called quasi-bosonic (or CS-boson) model. Many tests of the three-dimensional bosonization duality have been already performed, see e.g. [14, 15, 19-21]

AdS/CFT correspondence adds another direction to the diagram. Any of the four families of CFT's described above (there are actually only two families if the $3 d$ bosonization conjecture is true) is expected to have an $A d S_{4}$ dual. By the field content, the natural candidate dual is a HS theory. Indeed, in the strict $N=\infty$ limit each of the theories possesses an infinite number of conserved tensors. The coupling constant in dual HS theory is of order $1 / N$, while the t'Hooft constant $\lambda$ should be manifested as an additional coupling constant in the bulk theory. It is important to note that the Chern-Simons term breaks parity on the CFT side and therefore the dual HS theory should also violate parity unless we are at one of the limiting points. Remarkably, these are exactly the properties of the Vasiliev equations [22, 23]. There is a parameter that enters the equations in the form of a simple phase factor $e^{ \pm i \theta}$. For generic $\theta$ the equations do not preserve parity. There are two parity preserving options: $\theta=0$ and $\theta=\pi / 2$, which are called Type-A and Type-B [3]. On the $A d S_{4}$ side the difference between the duals of the CS-boson and CS-fermion models is in the boundary conditions for the scalar field of the HS multiplet. The relationships between the HS theories involved and their CFT duals are depicted in the diagram. The HS theories connected by the solid lines on the left (right) have $\theta=0(\theta=\pi / 2)$ and they differ from each other only with respect to the boundary conditions imposed on the scalar field present in HS theory. The upper (lower) solid lines denote the HS theory with generic $\theta$ parameter and $\Delta=1(\Delta=2)$ boundary conditions on the scalar field. 
Since we are going to study the AdS/CFT three-point functions, let us review the results obtained so far. In [11] the effects of parity breaking were studied to the lowest order in $1 / \tilde{N}$, but to all orders in $\tilde{\lambda}$. It was shown that the three point functions of HS currents $\left\langle j_{s} j_{s} j_{s}\right\rangle_{B, F}$ in CS-boson and CS-fermion theories decompose into three structures: two of them represent the free theories ('B' for CS-boson, 'F' for CS-fermion, ' $b$ ' for free boson and 'f' for free fermion) and another one is parity odd:

$$
\langle j j j\rangle_{B, F}=\tilde{N}\left[\cos ^{2} \theta\langle j j j\rangle_{b, f}+\sin ^{2} \theta\langle j j j\rangle_{f, b}+\cos \theta \sin \theta\langle j j j\rangle_{\text {odd }}\right],
$$

where $\cos ^{2} \theta=1 /\left(1+\tilde{\lambda}^{2}\right)$. At $\tilde{\lambda}=0$ the result simply states that the three-point functions are pure, i.e. are given by the free theory we started with. As we switch on the coupling there is an admixture of the odd structure and, with a bit suppressed factor, the contribution of the structure of the opposite free theory (free fermion for CS-boson and free boson for CS-fermion). In the strongly coupled regime everything is upside-down: the contribution of the starting free theory is suppressed, there is a parity-odd piece and the leading contribution is due to the opposite free theory. In the limit of the strong coupling the three-point functions are pure, but are those of the opposite theory. This transition manifests the bosonization duality. Scalar singlet operators require special treatment and are not captured by (1.1). In the first approximation the CS-boson theory has a scalar singlet operator $j_{0}$ of dimension 1 and the CS-fermion has a scalar singlet operator $\tilde{j}_{0}$ of dimension 2. Three-point functions with one insertion of $j_{0}\left(\tilde{j}_{0}\right)$ take a similar form given by [11]

$$
\begin{aligned}
\left\langle j_{s_{1}} j_{s_{2}} j_{0}\right\rangle_{B .} & =\tilde{N}\left[\cos \theta\left\langle j_{s_{1}} j_{s_{2}} j_{0}\right\rangle_{f . b .}+\sin \theta\left\langle j_{s_{1}} j_{s_{2}} j_{0}\right\rangle_{\text {odd }}\right] \\
\left\langle j_{s_{1}} j_{s_{2}} \tilde{j}_{0}\right\rangle_{F .} & =\tilde{N}\left[\cos \theta\left\langle j_{s_{1}} j_{s_{2}} \tilde{j}_{0}\right\rangle_{f . f .}+\sin \theta\left\langle j_{s_{1}} j_{s_{2}} \tilde{j}_{0}\right\rangle_{\text {odd }}\right] .
\end{aligned}
$$

and we discuss them in section 2.6. The difference is that with each $j_{0}\left(\tilde{j}_{0}\right)$ insertion we lose one structure. Another remarkable fact is that the $\tilde{\lambda}$-deformation appears as an overall factor of some structures as displayed above, i.e. there are no spin-dependent factors. Moreover, it was argued in [24] and then proved in [25] that all the structures are unique. For example, there is just one parity-odd structure $\left\langle j_{s} j_{s} j_{s}\right\rangle_{\text {odd }}$.

As far as the checks of the AdS/CFT duality are concerned, there exists a number of results. In [3] it was noted that the three-point coupling of the scalar field vanishes as obtained from the Vasiliev equation in the Schwinger-Fock gauge, which is consistent with vanishing of the three-point function in the critical vector model, corresponding to the $\Delta=2$ boundary condition on the bulk scalar. In the case of $\Delta=1$ boundary condition, however, a non-vanishing three point amplitude arises as a result of a regularization of a pertinent divergent integral [26-28], in agreement with the result from the free boson model. In [27] the first three-point functions were computed in the parity even case for two different spins $s_{1}, s_{2}$, the third one being zero (one scalar leg). It was assumed that the results give the correct CFT structures and it is possible to compare the leading coefficients only by choosing certain special kinematics. There are two types of terms in the HS equations that are relevant: we call them $\omega C$ (they are local) and $C C$ (may be non-local), which are explained in section 3. The computation in [27] was mostly based on $\omega C$ terms, which are local. We would like to point out that the $C C$-terms were found [27] to produce either divergent 
results or, after some regularization, the results that are inconsistent with CFT. In [12] it was noted that the $\omega C$-terms can produce parity-odd correlators. In particular, the $0-1-s$ parity-odd structure, which is fixed by conformal symmetry, was recovered in some limit. ${ }^{2}$

In [29] a different approach was adopted to compute amplitudes involving three different spins, which turns out to be equivalent, roughly speaking, to taking only the $C C$-terms into account. ${ }^{3}$ This approach exploits the extension of spacetime by spinorial coordinates. First of all, the $C C$-terms were found to yield a divergent result, which is consistent with the previous findings. Secondly, after some regularization in [29], the $C C$-terms give the parity-even correlators that are consistent with the duality. In fact, the regularization makes the $C C$-terms collapse onto the HS invariants, which are essentially the traces of the master fields in extended spacetime, or HS amplitudes [30-32]. These amplitudes are not represented as integrals of some vertex over the AdS space and for that reason they are not directly related to Witten diagrams. In fact, in this approach the amplitude computation amounts to a direct CFT computation without taking into account the local structure of AdS. However, this method, even if we accept the regularization, seems to lead to vanishing parity-odd correlators [29].

Going back to the approach of [27], in [33] the full structure of the second order corrections in the Vasiliev equations was worked out and it was shown that the resulting equations are too non-local to be treated by the field theory methods, which also explains infinities observed in [27, 29]. In [34] a modification of some of the equations was proposed to cure the problem at the second order.

In this paper we sharpen and expand considerably the tests of the HS holography discussed above. In particular we provide simplifications in the computation of HS correlation functions, and we establish nontrivial new tests of the holography for the parity violating HS theories. More specifically, our results can be summarized as follows. We isolate the scalar field equation in the Vasiliev theory up to quadratic terms in fields. This allows us to compute the $s_{1}-s_{2}-0$ correlators. The scalar field receives contribution only from $\omega C$ terms for $s_{1} \neq s_{2}$ and therefore does not rely on the structure of the $C C$-terms. The vertex is fixed by the HS algebra structure constants and also follows straightforwardly from the Vasiliev equations $[35,36]$. We evaluate the vertex on the boundary-to-bulk propagators, which results in a rather simple expression. Next we compute the bulk integral and recover the full structure of the correlation functions for the Chern-Simons matter theories, including the four limiting cases, namely the free/critical fermion/boson theories. The dependence on the parity violating parameter comes out right. For the parity-even cases our results furnish an improvement of [27] as the full structure is reproduced without assuming any special kinematics. ${ }^{4}$ The parity-violating structures we find from the bulk computations are compared with recent results [25] for Chern-Simons matter theories and found to be in a perfect agreement. As a byproduct we find explicit formulae for the parity violating structures (in [25] they were encoded in a system of recurrence relations for the coefficients).

\footnotetext{
${ }^{2}$ See (5.8) there for the AdS integral that is applicable for any $s_{1}, s_{2}$.

${ }^{3}$ The $\omega C$-terms seem to be missing, though there is a gauge choice ambiguity present in this method that could lead to such terms.

${ }^{4}$ In [27] a limit was taken such that the six conformally invariant structures that we review in the next section get reduced to just one structure.
} 
The correlators are analytic in spin and consequently the $s-s-0$ case can be reached too, as was already noted in [27], by evaluating the $s_{1}-s_{2}-0$ vertex for not equal spins and then setting $s_{1}=s_{2} .{ }^{5}$ This gives non-zero contribution even though the $\omega C$-term vanishes identically for $s_{1}=s_{2}$ and the full $s-s-0$ amplitude originates from the $C C$-term alone. We perform the simplest test of the modified equations proposed in [34] and recover the $s-s-0$ correlators with the right coefficient.

The outline of the paper is as follows. In section 2 we review the structure of the CFT correlators in Chern-Simons matter theories. In section 3 we discuss the general structure of HS interactions and relation to the Vasiliev equations. In section 4 we list the basic objects that constitute the boundary-to-bulk propagators for spinning fields. In section 5 the propagators for HS fields are given, and the vertex is evaluated on the propagators. The actual computation of the cubic Witten diagram is done in section 6 . In section 7 , we summarize and comment on our results, and we also discuss the contribution of the redefined $C C$ vertex [34] to the $s-s-0$ amplitude. Our notations and conventions are given in appendix A.

\section{CFT}

In this section we review the general structure of correlation function in three dimensions and list the results available in the literature for free theories and, more generally, for Chern-Simons matter theories. The advantage of three dimensions is that one can benefit from the fact that $s o(2,1) \sim s p(2)$.

\subsection{General structure of the correlators}

In three dimensions a traceless rank- $s$ so $(2,1)$-tensor is equivalent to a symmetric rank$2 s$ spin-tensor. It is convenient to contract the Lorentz indices of tensor operators with auxiliary polarization vectors that are now replaced by polarization spinors, which we denote by $\eta \equiv \eta^{\alpha}$. Therefore, a weight- $\Delta$, rank- $s$ tensor operator $O_{\Delta}^{a_{1} \ldots a_{s}}$ is replaced by a generating function $O_{\Delta}(\mathrm{x}, \eta):^{6}$

$$
O_{\Delta}^{a_{1} \ldots a_{s}}(\mathrm{x}) \quad \longrightarrow \quad O_{\Delta}^{\alpha_{1} \ldots \alpha_{2 s}}\left(\mathrm{x}^{\beta \gamma}\right) \quad \longrightarrow \quad O_{\Delta}(\mathrm{x}, \eta)=O_{\Delta}^{\alpha_{1} \ldots \alpha_{2 s}}(\mathrm{x}) \eta_{\alpha_{1}} \ldots \eta_{\alpha_{2 s}}
$$

Suppose we are given a number of operators $O\left(\mathrm{x}_{i}, \eta_{i}\right)$ that are inserted at points $\mathrm{x}_{i}$ and whose tensor indices are contracted with polarization spinors $\eta_{\alpha}^{i}$. The conformal group acts in the usual way. In particular, Lorentz transformations correspond to an $\mathrm{Sp}(2)$ matrix $A_{\alpha}{ }^{\beta}$ that acts both on coordinates $\mathrm{x}_{i}$ and polarization spinors $\eta^{i}$ :

$$
\mathrm{x}^{\beta \delta} \rightarrow A_{\alpha}{ }^{\beta} A_{\gamma}{ }^{\delta} \mathrm{x}^{\alpha \gamma}, \quad \eta_{\alpha}^{i} \rightarrow A_{\alpha}{ }^{\beta} \eta_{\beta}^{i} \text {. }
$$

\footnotetext{
${ }^{5}$ In [12], the $s-s-0$ bulk integral was shown to reproduce the generating function for even and odd correlators found in [5]. This generating function should give the right conformal structures whose normalization is yet to be fixed by comparing with CS-matter theories.

${ }^{6} 3 d$ coordinates $\vec{x}$ are replaced by two-by-two symmetric matrices $\mathrm{x}^{\alpha \beta}$. For further notations and conventions see appendix A.
} 
It is useful to define the inversion map as

$$
R \overrightarrow{\mathrm{x}}=\frac{\overrightarrow{\mathrm{x}}}{\mathrm{x}^{2}}, \quad R \eta_{\alpha}^{a}=\frac{\mathrm{x}_{\alpha}{ }^{\beta} \eta_{\beta}^{a}}{\mathrm{x}^{2}}, \quad R x^{\alpha \dot{\alpha}}=\frac{x^{\alpha \dot{\alpha}}}{x^{2}}=\frac{\mathrm{x}^{\alpha \dot{\alpha}}+i z \epsilon^{\alpha \dot{\alpha}}}{\mathrm{x}^{2}+z^{2}}
$$

Then, it is not hard to see that the following structures are conformally invariant [24]:

$$
\begin{aligned}
P_{i j} & =\eta_{\alpha}^{i} R\left[\mathrm{x}_{i}-\mathrm{x}_{j}\right]^{\alpha \beta} \eta_{\beta}^{j}, & R P_{i j} & =P_{i j}, \\
Q_{j k}^{i} & =\eta_{\alpha}^{i}\left(R\left[\mathrm{x}_{j}-\mathrm{x}_{i}\right]-R\left[\mathrm{x}_{k}-\mathrm{x}_{i}\right]\right)^{\alpha \beta} \eta_{\beta}^{i}, & R Q_{j k}^{i} & =Q_{j k}^{i} .
\end{aligned}
$$

There is also one more structure that is parity-odd:

$$
S_{j k}^{i}=\frac{\eta_{\alpha}^{k}\left(\mathrm{x}_{k i}\right)^{\alpha}{ }_{\beta}\left(\mathrm{x}_{i j}\right)^{\beta \gamma} \eta_{\gamma}^{j}}{\mathrm{x}_{i j} \mathrm{x}_{i k} \mathrm{x}_{j k}}, \quad R S_{j k}^{i}=-S_{j k}^{i}
$$

Any three-point correlation function $\left\langle O_{1}\left(\mathrm{x}_{1}, \eta^{1}\right) O_{2}\left(\mathrm{x}_{2}, \eta^{2}\right) O_{3}\left(\mathrm{x}_{3}, \eta^{3}\right)\right\rangle$ can be decomposed into an obvious prefactor times a polynomial in $Q, P, S$ structures:

$$
\left\langle O_{1}\left(\mathrm{x}_{1}, \eta^{1}\right) O_{2}\left(\mathrm{x}_{2}, \eta^{2}\right) O_{3}\left(\mathrm{x}_{3}, \eta^{3}\right)\right\rangle=\frac{1}{\mathrm{x}_{12}^{\Delta_{1}+\Delta_{2}-\Delta_{3}} \mathrm{x}_{13}^{\Delta_{1}+\Delta_{3}-\Delta_{2}} \mathrm{x}_{23}^{\Delta_{2}+\Delta_{3}-\Delta_{1}}} f(P, Q, S) .
$$

The function $f$ must comply with the spin of the operators and also should not contain any redundant structures that are possible due to not all of $Q, P, S$ being independent. As we will need only two- and three-point correlation functions, it is convenient to introduce the following notation

$$
Q_{1} \equiv Q_{32}^{1}, \quad Q_{2} \equiv Q_{13}^{2}, \quad Q_{3} \equiv Q_{21}^{3}, \quad S_{3} \equiv S_{21}^{3} \text {. }
$$

The even power of any odd structure is even, which is manifested by [24]

$$
S_{3}^{2}+Q_{1} Q_{2}-P_{12}^{2} \equiv 0
$$

This is the only relation we need for the $s_{1}-s_{2}-0$ correlators. The even structures $P, Q$ can be identified as the building blocks of the simplest correlators that are completely fixed by conformal symmetry

$$
\begin{aligned}
\left\langle j_{s_{1}}\left(\mathrm{x}_{1}, \eta_{1}\right) j_{s_{2}}\left(\mathrm{x}_{2}, \eta_{2}\right)\right\rangle & \sim \frac{1}{\mathrm{x}_{12}^{2}} \delta_{s_{1}, s_{2}}\left(P_{12}\right)^{s_{1}+s_{2}}, \\
\left\langle j_{s_{1}}\left(\mathrm{x}_{1}, \eta_{1}\right) j_{0}\left(\mathrm{x}_{2}\right) j_{0}\left(\mathrm{x}_{3}\right)\right\rangle & \sim \frac{1}{\mathrm{x}_{12} \mathrm{x}_{23} \mathrm{x}_{31}}\left(Q_{1}\right)^{s_{1}},
\end{aligned}
$$

where we assumed that $j_{s}$ is the spin- $s$ conserved tensor and the weight of scalar operator $j_{0}$ is $\Delta=1$. The conservation of currents can be imposed with the help of a simple third order operator:

$$
\operatorname{div}=\frac{\partial^{2}}{\partial \eta_{\alpha} \partial \eta_{\beta}} \frac{\partial}{\partial \mathrm{x}^{\alpha \beta}}
$$




\subsection{Free boson}

The simplest example of duality is between the (non)-minimal the Type-A HS gravity and $(\mathrm{U}(N)) O(N)$ free scalars. Dropping the canonical normalization factors, the two-point functions for the $\mathrm{U}(N)$ case $\operatorname{are}^{7}$

$$
\mathrm{U}(N): \quad\left\langle\bar{\phi}_{a}(\mathrm{x}) \phi^{b}(0)\right\rangle=\delta_{a}^{b} \frac{1}{|\mathrm{x}|},
$$

where $a, b=1, \ldots, N$. As is well known $[27,37,38]$, it is convenient to pack the HS currents into generating functions

$$
j(\mathrm{x}, \eta)=\left.f(u, v) \bar{\phi}_{a}\left(\mathrm{x}_{1}\right) \phi^{a}\left(\mathrm{x}_{2}\right)\right|_{\mathrm{x}_{i}=\mathrm{x}}, \quad u=\frac{1}{2} \eta^{\alpha} \eta^{\beta} \partial_{\alpha \beta}^{1}, \quad v=\frac{1}{2} \eta^{\alpha} \eta^{\beta} \partial_{\alpha \beta}^{2} .
$$

The conservation of the current implies a simple differential equation for $f$. The most convenient formulae are obtained $[38,39]$ with the help of an auxiliary generating function $C^{a}(\eta \mid x)$ for the derivatives of the scalar field. It obeys $\partial_{\alpha \beta} C^{a}=\frac{i}{2} \partial_{\alpha} \partial_{\beta} C^{a}$, which is solved by $C^{a}=\cos \left[2 e^{i \pi / 4} \sqrt{u}\right] \phi^{a}(\mathrm{x}){ }^{8}$ The generating function of the HS currents then has a simple factorized form: ${ }^{9}$

$$
j(\mathrm{x}, \eta)=C_{a}(u) C^{a}(-v)=\left.\cos \left[2 e^{i \pi / 4} \sqrt{u}\right] \cos \left[2 e^{i \pi / 4} \sqrt{-v}\right] \bar{\phi}_{a}\left(\mathrm{x}_{1}\right) \phi^{a}\left(\mathrm{x}_{2}\right)\right|_{\mathrm{x}_{i}=\mathrm{x}} .
$$

The two-point function of the currents is then ${ }^{10}$

$$
\left\langle j_{s} j_{s}\right\rangle_{f . b .} \equiv\left\langle j\left(\mathrm{x}_{1}, \eta_{1}\right) j\left(\mathrm{x}_{2}, \eta_{2}\right)\right\rangle=\frac{1}{2} \frac{1}{\mathrm{x}_{12}^{2}} \cosh \left(2 P_{12}\right),
$$

where we introduced a shorthand notation $j_{s}$ for $j\left(\mathrm{x}_{i}, \eta_{i}\right)$ and the arguments are in accordance with its position inside the brackets; f.b. refers to "free boson". Sometimes we have to distinguish the first member of the family $j_{0} \equiv \bar{\phi}_{a}(\mathrm{x}) \phi^{a}(\mathrm{x})$ from the others. The simplest three-point function, which is fixed by the symmetry, is

$$
\left\langle j_{s} j_{0} j_{0}\right\rangle_{f . b .}=\frac{2}{\mathrm{x}_{12} \mathrm{x}_{13} \mathrm{x}_{23}} \cos \left(\frac{1}{2} Q_{1}\right) .
$$

The three-point functions with two HS currents are assembled into

$$
\left\langle j_{s} j_{s} j_{0}\right\rangle_{f . b .}=\frac{2}{\mathrm{x}_{12} \mathrm{x}_{13} \mathrm{x}_{23}} \cos \left(\frac{1}{2} Q_{1}+\frac{1}{2} Q_{2}\right) \cos P_{12} .
$$

\footnotetext{
${ }^{7}$ In the $O(N)$ case we omit the bar over $\phi$, i.e. we have $\phi^{a}$, and the $\mathrm{U}(N)$-invariant tensor on the r.h.s. $\delta_{b}^{a}$ is replaced by the $O(N)$-invariant one $\delta^{a b}$.

${ }^{8}$ One can put any factor $k$ into the definition $\partial_{\alpha \beta} C^{a}=k \partial_{\alpha} \partial_{\beta} C^{a}$, which then will alter the normalization of the correlators. Our factors are such as to facilitate the comparison on the both sides of AdS/CFT duality.

${ }^{9}$ There is another generating function that can be obtained directly from the conservation equation $\left(\partial_{u}+\partial_{v}+2 u \partial_{u}^{2}+2 v \partial_{v}^{2}\right) f=0$, which is $f=e^{u-v} \cos (2 \sqrt{u v})[27]$.

${ }^{10}$ There is always an overall factor of $N$, which is present in all two-point and three-point functions of the HS currents. We will suppress this factor. Correlation function for specific spins $s_{1}, s_{2}, \ldots$ is extracted out of any generating function as the coefficient of $\left(\eta_{1}\right)^{s_{1}}\left(\eta_{2}\right)^{s_{2}} \ldots$.
} 
The three-point functions of the three HS currents are [29] (see also [24, 31, 32, 39, 40]):

$$
\left\langle j_{s} j_{s} j_{s}\right\rangle_{f . b .}=\frac{2}{\mathrm{x}_{12} \mathrm{x}_{23} \mathrm{x}_{31}} \cos \left(\frac{1}{2} Q_{1}+\frac{1}{2} Q_{2}+\frac{1}{2} Q_{3}\right) \cos \left(P_{12}\right) \cos \left(P_{23}\right) \cos \left(P_{31}\right) .
$$

It is also useful to consider the case of $\mathrm{U}(N)$-singlet constraint with leftover $\mathrm{U}(M)$ global symmetry. The generating function is

$$
\left\langle j_{s} j_{s} j_{0}\right\rangle_{f . b .}=\frac{2}{\mathrm{x}_{12} \mathrm{x}_{13} \mathrm{x}_{23}} \exp \left[-\frac{i}{2}\left(Q_{1}+Q_{2}\right)\right] \cos P_{12} .
$$

This is the most general case, all others being simple truncations. Imposing the bose symmetry we get (2.18). Truncation to even spins only gives the $O(N)$ case.

\subsection{Free fermion}

The second example is the duality of the Type-B theory and a theory of free $\mathrm{U}(N)$ or $\operatorname{USp}(N)$ fermions, of which we consider the former. The two-point function is

$$
\mathrm{U}(N): \quad\left\langle\bar{\psi}_{a \alpha}(\mathrm{x}) \psi_{\beta}^{b}(0)\right\rangle=\delta_{a}^{b} \frac{1}{2} \frac{\overrightarrow{\mathrm{x}} \cdot \vec{\sigma}_{\alpha \beta}}{|\mathrm{x}|^{3}}=\delta_{a}^{b} \partial_{\alpha \beta}\left|\mathrm{x}^{2}\right|^{-\frac{1}{2}}
$$

HS currents are constructed analogously

$$
j(\eta, \mathrm{x})=\left.f(u, v) \eta^{\alpha} \eta^{\beta} \psi_{\alpha}^{a}\left(\mathrm{x}_{1}\right) \psi_{a \beta}\left(\mathrm{x}_{2}\right)\right|_{\mathrm{x}_{i}=\mathrm{x}},
$$

and again the useful trick is to pack the derivatives into $C^{a}$ as follows

$$
\partial_{\alpha \beta} C^{a}=\frac{i}{2} \partial_{\alpha} \partial_{\beta} C^{a}, \quad \quad C^{a}=\frac{1}{\sqrt{u}} \sin \left[2 e^{i \pi / 4} \sqrt{u}\right] \psi_{\alpha}^{a}(\mathrm{x}) \eta^{\alpha} .
$$

The generating function of the HS currents has the factorized form:

$$
j(\eta, \mathrm{x})=C^{a}(u) C_{a}(-v)=\left.\frac{1}{\sqrt{-u v}} \sin \left[2 e^{i \pi / 4} \sqrt{u}\right] \sin \left[2 e^{i \pi / 4} \sqrt{-v}\right] \psi_{\alpha}^{a}\left(\mathrm{x}_{1}\right) \psi_{a \beta}\left(\mathrm{x}_{2}\right)\right|_{\mathrm{x}_{i}=\mathrm{x}} .
$$

The two-point function is normalized in the same way as that of the free boson:

$$
\left\langle j_{s} j_{s}\right\rangle_{f . f .}=\frac{1}{2} \frac{1}{\mathrm{x}_{12}^{2}} \cosh \left(2 P_{12}\right), \quad\left\langle\tilde{j}_{0} \tilde{j}_{0}\right\rangle_{f . f .}=\frac{1}{4 \mathrm{x}^{4}}
$$

where $f . f$. refers to "free fermion". Here the scalar singlet operator

$$
\tilde{j}_{0}=\bar{\psi}_{a} \psi^{a}
$$

has dimension 2 and is not captured by the generating function above. The three-point function of $\tilde{j}_{0}$ vanishes due to parity

$$
\left\langle\tilde{j}_{0} \tilde{j}_{0} \tilde{j}_{0}\right\rangle_{f . f .}=0 .
$$


For the other cases we find (see also $[24,31,32,39,40]$ ):

$$
\begin{aligned}
& \left\langle j_{s} j_{s} \tilde{j}_{0}\right\rangle_{f . f .}=\frac{2 \cos \left(\frac{1}{2} Q_{1}+\frac{1}{2} Q_{2}\right)}{\mathrm{x}_{23}^{2} \mathrm{x}_{13}^{2}} S_{3} \sin P_{12}, \\
& \left\langle j_{s} \tilde{j}_{0} \tilde{j}_{0}\right\rangle_{f . f .}=\frac{2 \sin \frac{1}{2} Q_{1}}{\mathrm{x}_{12} \mathrm{x}_{23}^{3} \mathrm{x}_{31}} Q_{1},
\end{aligned}
$$

and

$$
\left\langle j_{s} j_{s} j_{s}\right\rangle_{f . f .}=\frac{2}{\mathrm{x}_{12} \mathrm{x}_{23} \mathrm{x}_{31}} \sin \left(\frac{1}{2} Q_{1}+\frac{1}{2} Q_{2}+\frac{1}{2} Q_{3}\right) \sin \left(P_{12}\right) \sin \left(P_{23}\right) \sin \left(P_{31}\right) .
$$

Note that the expression for the correlators of $j_{s}$ is valid for $s \geq 1$ only, while any insertion of $\tilde{j}_{0}$ should be treated separately. Again, it is useful to work with the free fermion with leftover $\mathrm{U}(M)$ global symmetry and the generating function is

$$
\left\langle j_{s} j_{s} \tilde{j}_{0}\right\rangle_{f . f .}=\frac{2}{\mathrm{x}_{23}^{2} \mathrm{x}_{13}^{2}} \exp \left(\frac{i}{2} Q_{1}+\frac{i}{2} Q_{2}\right) S_{3} \sin P_{12}
$$

\subsection{Critical boson}

Critical Boson theory is the IR fixed point under a double-trace deformation $\left(\phi^{2}\right)^{2}$. The regime that is relevant for AdS/CFT is the large- $N$, see e.g. [41, 42] for the systematic $1 / N$ expansion. To the leading order in $1 / N$ the correlation function of HS currents $j_{s}, s>0$ stays the same as in the free boson theory, i.e. (2.19). The dimension of the lowest singlet operator $\phi^{2}$, which is usually dubbed $\sigma$, jumps from 1 for free boson to $2+\mathcal{O}(1 / N)$ for critical one. Therefore, the spectrum of the singlet operators in the critical boson theory looks like that of the free fermion in $N=\infty$ limit. For this reason the scalar singlet is denoted as $\tilde{j}_{0}$. Correlation functions with a number of $\tilde{j}_{0}$ insertions are related to those in the free boson theory by attaching propagators of the $\sigma$-field. In [25] the three-point functions $\left\langle j_{s_{1}} j_{s_{2}} \tilde{j}_{0}\right\rangle$ were fixed by employing the non-conservation equation in the ChernSimons matter theories. The result is ${ }^{11}$

$$
\left\langle j_{s_{1}} j_{s_{2}} \tilde{j}_{0}\right\rangle_{c . b .}=f_{s_{1}, s_{2}} \frac{1}{\mathrm{x}_{13}^{2} \mathrm{x}_{23}^{2}}\left(Q_{1}\right)^{s_{1}}\left(Q_{2}\right)^{s_{2}} \sum_{k} A_{k}\left(\frac{P_{12}^{2}}{Q_{1} Q_{2}}\right)^{k}
$$

where $c . b$. refers to "critical boson", $f_{s_{1}, s_{2}}$ is a overall spin-dependent factor and the coefficients obey $A_{-1}=0, A_{0}=1$ and the rest are generated via

$$
A_{n}=\frac{\left.A_{n-1}\left(s_{1} s_{2}+\left(2 n-1-s_{1}\right)\left(2 n-1-s_{2}\right)-5 n+4\right)\right)-2 A_{n-2}\left(n-s_{1}-2\right)\left(n-s_{2}-2\right)}{n(2 n-1)} .
$$

The coefficients $f_{s_{1}, s_{2}}$ depend on normalization of $\left\langle j_{s} j_{s}\right\rangle_{c . b .}$. two-point functions. We choose the same normalization as in the free boson theory (2.16), which gives:

$$
f_{s_{1}, s_{2}}=-\frac{i^{s_{1}+s_{2}} 2^{-s_{1}-s_{2}+2}}{\Gamma\left(s_{1}+\frac{1}{2}\right) \Gamma\left(s_{2}+\frac{1}{2}\right)} .
$$

\footnotetext{
${ }^{11}$ We simplify a bit the form of the result by noticing that the solution to the recurrence relations is easier to obtain starting from the last coefficients as compared to [25].
} 


\subsection{Critical fermion}

Critical fermion is the Gross-Neveu model, i.e. free fermion with $(\bar{\psi} \psi)^{2}$-deformation added. The large- $N$ expansion works fine despite the apparent non-renormalizability of the interactions, see e.g. [43, 44]. Again, the correlation functions of HS currents $j_{s}, s>0$ are the same as in the free fermion theory $(2.30)$ to the leading order in $1 / N$, while the dimension of $\sigma=\bar{\psi} \psi$ jumps from 2 in the free theory to $1+\mathcal{O}(1 / N)$ in the Gross-Neveu one. Therefore, the spectrum of singlet operators looks like that of the free boson theory in the $N=\infty$ limit and we use the same notation $j_{0}$ for $\bar{\psi} \psi$. The three-point functions $\left\langle j_{s_{1}} j_{s_{2}} j_{0}\right\rangle$ were found in [25] to have the form:

$$
\left\langle j_{s_{1}} j_{s_{2}} j_{0}\right\rangle_{c . f .}=g_{s_{1}, s_{2}} \frac{1}{\mathrm{x}_{13} \mathrm{x}_{23} \mathrm{x}_{12}} S_{3} P_{12}\left(Q_{1}\right)^{s_{1}-1}\left(Q_{2}\right)^{s_{2}-1} \sum_{k} A_{k}\left(\frac{P_{12}^{2}}{Q_{1} Q_{2}}\right)^{k}
$$

where c.f. refers to "critical fermion", $A_{-1}=0, A_{0}=1$ and the rest of $A_{k}$ is generated via

$$
A_{n}=-\frac{8 A_{n-2}\left(-n+s_{1}+1\right)\left(-n+s_{2}+1\right)-4 A_{n-1}\left(\left(2 n-s_{1}-1\right)\left(2 n-s_{2}-1\right)+n+s_{1} s_{2}\right)}{4 n(2 n+1)} .
$$

The overall factor in our normalization is

$$
g_{s_{1}, s_{2}}=-\frac{i^{s_{1}+s_{2}} 2^{-s_{1}-s_{2}+2}}{\Gamma\left(s_{1}+\frac{1}{2}\right) \Gamma\left(s_{2}+\frac{1}{2}\right)} .
$$

\subsection{Chern-Simons matter theories}

There are four theories that are obtained by coupling the free/critical boson/fermion theories discussed above to Chern-Simons gauge field. The conjecture of three-dimensional bosonization is that they are equivalent in pairs under an appropriate identification of parameters $N$ and $\lambda=N / k$. We are interested in $\left\langle j_{s_{1}} j_{s_{2}} j_{0}\right\rangle$ and $\left\langle j_{s_{1}} j_{s_{2}} \tilde{j}_{0}\right\rangle$ correlators, which are constrained to be [11]: ${ }^{12}$

$$
\begin{aligned}
\left\langle j_{s_{1}} j_{s_{2}} j_{0}\right\rangle_{B .} & =\tilde{N}\left[\cos \theta\left\langle j_{s_{1}} j_{s_{2}} j_{0}\right\rangle_{f . b .}+\sin \theta\left\langle j_{s_{1}} j_{s_{2}} j_{0}\right\rangle_{\text {odd }}\right] \\
\left\langle j_{s_{1}} j_{s_{2}} \tilde{j}_{0}\right\rangle_{F .} & =\tilde{N}\left[\cos \theta\left\langle j_{s_{1}} j_{s_{2}} \tilde{j}_{0}\right\rangle_{f . f .}+\sin \theta\left\langle j_{s_{1}} j_{s_{2}} \tilde{j}_{0}\right\rangle_{\text {odd }}\right],
\end{aligned}
$$

where $B$. and $F$. refer to CS-boson and CS-fermion, respectively. Here $\tilde{N}$ and $\theta$ are two macroscopical parameters and we recall that $\cos ^{2} \theta=1 /\left(1+\tilde{\lambda}^{2}\right)$. This result holds true to the leading order in $\tilde{N}$ but to all orders in $\tilde{\lambda}$. The expressions in terms of the microscopical parameters depend on type of theory and on $N, \lambda=N / k$. We can work with $\tilde{N}$ and $\theta$ since their microscopical origin is invisible from the bulk. Note that the odd structures are different in the two cases (they even have different conformal dimensions due to $j_{0}$ and $\tilde{j}_{0}$ ). In fact the two odd structures can be found as the regular correlators on the opposite side of the bosonization duality:

$$
\left\langle j_{s_{1}} j_{s_{2}} j_{0}\right\rangle_{\text {odd }}=\left\langle j_{s_{1}} j_{s_{2}} j_{0}\right\rangle_{c . f .}, \quad\left\langle j_{s_{1}} j_{s_{2}} \tilde{j}_{0}\right\rangle_{\text {odd }}=\left\langle j_{s_{1}} j_{s_{2}} \tilde{j}_{0}\right\rangle_{c . b .} .
$$

\footnotetext{
${ }^{12}$ There is some ambiguity in the overall factor due to the choice of normalization of the scalar singlet operator.
} 
As far as $\left\langle j_{s_{1}} j_{s_{2}} \tilde{j}_{0}\right\rangle$ correlators are concerned, all the results reviewed in the previous sections follow from (2.36) and this is the structure we would like to reproduce from the bulk side, including the details of the correlators and normalization factors. Note that the term 'parity odd correlator' corresponding to the second part of (2.36) may not have anything to do with parity, in view of (2.37). For example, the usual parity even correlator $\left\langle j_{s} j_{s} \tilde{j}_{0}\right\rangle_{c . b}$. in the critical boson theory appears to be odd from the point of view of the dual fermionic theory.

\section{$3 \quad$ Higher spin interactions}

We briefly review the structure of the equations that results from the Vasiliev equations [35]. For more detailed reviews, we refer to $[13,23,45]$. The corrections to the free equations that are bilinear in the fields were worked out in $[27,33,36,45]$. The main conclusion that is based on $[27,33]$ is that, up to quadratic order, the Klein-Gordon equation is sourced by two type of terms. One part is fixed by the HS algebra and is local enough for the computation of the correlation functions using field theory tools. A second part gives rise to infinities, though a proposal has been made [34] on how to obtain finite results by a set of field redefinitions. While our computations will mostly be based on the use of the first part, we shall nonetheless test this proposal as well in section 5, and comment further about it in section 7 .

The convenient field variables that a HS theory can be built with are the Fronsdal fields. The Vasiliev equations yield first order differential equations, which upon solving for the auxiliary fields give equations in term of the Fronsdal fields and an infinitely many of differential consequences of these equations, which we drop.

For the purpose of computing tree-level Witten diagrams it is sufficient to impose the transverse and traceless gauge. Then the $4 d$ free Fronsdal equations for spins $s \geq 1$ read: ${ }^{13}$

$$
\left(\square+2\left(s^{2}-2 s-2\right)\right) \Phi_{\underline{m}_{1} \ldots \underline{m}_{s}}=0, \quad \Phi^{\underline{n}} \underline{n m}_{3} \ldots \underline{m}_{s}=0, \quad \nabla \underline{n}^{n} \Phi_{\underline{n} m_{2} \ldots \underline{m}_{s}}=0 .
$$

To make a link to the frame-like and then to the unfolded formulation of the $4 d$ HS theories we replace a set of the traceless world tensors $\Phi_{\underline{m}_{1} \ldots \underline{m}_{s}}$ with the generating function in the spinorial language as ${ }^{14}$

$$
\Phi(y, \bar{y} \mid x)=\sum_{s} \frac{1}{s ! s !} \Phi_{\alpha_{1} \ldots \alpha_{s}, \dot{\alpha}_{1} \ldots \dot{\alpha}_{s}}(x) y^{\alpha_{1}} \ldots y^{\alpha_{s}} \bar{y}^{\dot{\alpha}_{1}} \ldots \bar{y}^{\dot{\alpha}_{s}} .
$$

Then the gauge-fixed Fronsdal equations, as recovered from the $4 d$ HS theory, read:

$$
\left(\square+2\left(N^{2}-2 N-2\right)\right) \Phi=0, \quad(\partial \nabla \bar{\partial}) \Phi=0,
$$

where $N$ is the number operator $N=y^{\alpha} \partial_{\alpha}$ that counts spin (equivalently we can use $\left.\bar{N}=\bar{y}^{\dot{\alpha}} \partial_{\dot{\alpha}}\right)$. The last equation manifests the transverse gauge.

\footnotetext{
${ }^{13}$ Our normalization of the cosmological constant differs by a factor of two from the canonical one, which is the price to pay for the most natural coefficients in the $s p(4)$-flatness condition that provides us with the vierbein and spin-connection of $A d S_{4}$. See appendix A for further details.

${ }^{14}$ We note that the Fronsdal field is now a fiber (spin)-tensor, which is the world one contracted with $s$ vierbeins.
} 
In the unfolded approach [46], a specific multiplet of HS fields is packed into generating functions $\omega$ and $C$ that take values in a HS algebra. In our case the relevant HS algebra [47] is the even part of the Weyl algebra $A_{2}$. It is convenient to split the four generators of $A_{2}$ into (anti)-fundamentals of $\operatorname{sl}(2, \mathbb{C})$

$$
\left[\hat{y}_{\alpha}, \hat{y}_{\beta}\right]=2 i \epsilon_{\alpha \beta}, \quad\left[\hat{\bar{y}}_{\dot{\alpha}}, \hat{\bar{y}}_{\dot{\beta}}\right]=2 i \epsilon_{\dot{\alpha} \dot{\beta}}
$$

In practice, non-commuting operators $\hat{y}_{\alpha}$ and $\hat{\bar{y}}_{\dot{\alpha}}$ are replaced by commuting variables $y_{\alpha}$, $\bar{y}_{\dot{\alpha}}$ while the product is replaced with the star product

$$
(f \star g)(y, \bar{y})=f(y, \bar{y}) \exp i\left[\frac{\overleftarrow{\partial}}{\partial y^{\alpha}} \epsilon^{\alpha \beta} \frac{\vec{\partial}}{\partial y^{\beta}}+\frac{\overleftarrow{\partial}}{\partial \bar{y}^{\dot{\alpha}}} \epsilon^{\dot{\alpha} \dot{\beta}} \frac{\vec{\partial}}{\partial \bar{y}^{\dot{\beta}}}\right] g(y, \bar{y})
$$

The bosonic higher spin algebra is defined as the even subalgebra, i.e. $f(y, \bar{y}) \in h s$ implies $f(y, \bar{y})=f(-y,-\bar{y})$. It is straightforward to tensor any higher spin algebra with matrix algebra as to get $\mathrm{U}(N)$ extension, for example. The SUSY case are studied in [48].

The field content consists of one-form $\omega=\omega_{\underline{m}}(y, \bar{y} \mid x) d x^{\underline{m}}$ and zero-form $C=C(y, \bar{y} \mid x)$. In free theory the Fronsdal fields can be identified with certain components of $\omega$, which in the traceless gauge is

$$
h^{\alpha \dot{\alpha}} \partial_{\alpha} \partial_{\dot{\alpha}} \Phi(y, \bar{y} \mid x) \in \omega(y, \bar{y} \mid x)
$$

$\omega$ and $C$ obey the following linearized equations, known as the on mass-shell theorem (OMST) [46],

$$
\mathrm{D} \omega=\mathcal{V}(\Omega, \Omega, C), \quad \widetilde{\mathrm{D}} C=0,
$$

where $\mathcal{V}(\Omega, \Omega, C)$ is a star function quadratic in $\Omega$ and linear in $C$, and

$$
\begin{aligned}
& \mathrm{D} \omega \equiv d \omega-\Omega \star \omega-\omega \star \Omega=\nabla \omega-h^{\alpha \dot{\alpha}}\left(y_{\alpha} \bar{\partial}_{\dot{\alpha}}+\bar{y}_{\dot{\alpha}} \partial_{\alpha}\right) \omega, \\
& \widetilde{\mathrm{D}} C \equiv d C-\Omega \star C+C \star \pi(\Omega)=\nabla C+i h^{\alpha \dot{\alpha}}\left(y_{\alpha} \bar{y}_{\dot{\alpha}}-\partial_{\alpha} \bar{\partial}_{\dot{\alpha}}\right) C .
\end{aligned}
$$

The $A d S_{4}$ connection $\Omega$ is defined in appendix $\mathrm{A}$, and the Lorentz covariant derivative acts in the same way on $\omega(y, \bar{y} \mid x)$ and $C(y, \bar{y} \mid x)$ as

$$
\nabla \equiv d-\omega_{(0)}^{\alpha \beta} y_{\alpha} \partial_{\beta}-\bar{\omega}_{(0)}^{\dot{\alpha} \dot{\beta}} \bar{y}_{\dot{\alpha}} \bar{\partial}_{\dot{\beta}}
$$

The difference between $\mathrm{D}$ and $\widetilde{\mathrm{D}}$ is due to automorphism $\pi: \pi(f)(y, \bar{y})=f(y,-\bar{y})=$ $f(-y, \bar{y})$. The vertex that relates the order-s curl of the Fronsdal field to the HS Weyl tensors reads

$$
\mathcal{V}(\Omega, \Omega, C)=A\left[H^{\alpha \beta} \partial_{\alpha} \partial_{\beta} C(y, 0) e^{-i \theta}+\bar{H}^{\dot{\alpha} \dot{\beta}} \bar{\partial}_{\dot{\alpha}} \bar{\partial}_{\dot{\beta}} C(0, \bar{y}) e^{i \theta}\right],
$$

where $H^{\alpha \beta}=h_{\gamma}^{\alpha} \wedge h^{\beta \gamma}$, and analogously for $\bar{H}^{\dot{\alpha} \dot{\beta}}$. The constant $A$ is an arbitrary normalization factor that we choose to be $A=i / 4$. The linearized equations (3.7) are invariant under the linearized HS gauge transformations

$$
\delta \omega=\mathrm{D} \xi, \quad \delta C=0
$$


Equations (3.7) are equivalent to Fronsdal equations supplemented with differential consequences thereof. Up to the second order the unfolded equations should have the following schematic form

$$
\begin{aligned}
& d \omega=\omega \star \omega+\mathcal{V}(\omega, \omega, C)+\mathcal{V}^{2}(\omega, \omega, C, C)+\mathcal{O}\left(C^{3}\right), \\
& d C=\omega \star C-C \star \pi(\omega)+\mathcal{U}(\omega, C, C)+\mathcal{O}\left(C^{3}\right),
\end{aligned}
$$

where the vertices $\mathcal{V}^{2}$ and $\mathcal{U}$ need to be specified. The free equations result upon substituting $\omega \rightarrow \Omega+\omega$ and picking the terms that are linear in $\omega$ and $C$ :

$$
\mathrm{D} \omega^{(1)}=\mathcal{V}\left(\Omega, \Omega, C^{(1)}\right), \quad \widetilde{\mathrm{D}} C^{(1)}=0 .
$$

At the second order the weak-field expansion over the AdS background leads to

$$
\begin{aligned}
\mathrm{D} \omega^{(2)}-\mathcal{V}\left(\Omega, \Omega, C^{(2)}\right) & =\omega^{(1)} \star \omega^{(1)}+2 \mathcal{V}\left(\Omega, \omega^{(1)}, C^{(1)}\right)+\mathcal{V}\left(\Omega, \Omega, C^{(1)}, C^{(1)}\right), \\
\widetilde{\mathrm{D}} C^{(2)} & =\omega^{(1)} \star C^{(1)}-C^{(1)} \star \pi\left(\omega^{(1)}\right)+\mathcal{U}\left(\Omega, C^{(1)}, C^{(1)}\right) .
\end{aligned}
$$

We see that the second order fluctuations are sourced by the terms that are bilinear in the first order fluctuations. The terms that are bilinear in the zero-forms, the $C C$-terms for short, can be non-local. The part of the equations that does not have any problems with locality comes from the commutator in the HS algebra

$$
\widetilde{\mathrm{D}} C^{(2)}=\omega^{(1)} \star C^{(1)}-C^{(1)} \star \pi\left(\omega^{(1)}\right)+\mathcal{O}\left(C^{2}\right) .
$$

For some values of spins we do not expect the $C C$-terms to contribute. Indeed, when one of the legs is scalar, there is a unique (parity-preserving) coupling $s_{1}-s_{2}-0$, [49]. It is non-abelian whenever $s_{1} \neq s_{2}$ and is abelian for $s_{1}=s_{2}$.

Let us note that the computation of correlators from the equations of motion that are not derived from an action principle does not guarantee the bose symmetry of the correlators under the permutation of the points. The same correlator $\left\langle j_{s_{1}} j_{s_{2}} j_{0}\right\rangle$ can be obtained in two different ways: either by treating $j_{s_{1}}, j_{s_{2}}$ as sources and then solving for the scalar field or by treating $j_{s_{1}}, j_{0}$ as sources and solving for the spin- $s_{2}$ Weyl tensor. Both computations are possible with (3.16). It was checked in [27] for the leading coefficients that the two ways of getting the same correlator give a bose symmetric correlator.

Lastly, eq.(3.16) remains consistent if the fields $\omega, C$ are extended to matrix-valued fields. This way one can introduce Yang-Mills groups on top of the HS algebra. On the CFT side this should correspond to leftover global symmetries, i.e. those symmetries that remain after the singlet constraint is imposed.

\section{Kinematics of the boundary-to-bulk propagators}

In this section we discuss the boundary-to-bulk propagators for HS fields. The problem has been extensively studied starting from the lower spin fields, see e.g. [50]. Specifically, we need the unfolded propagators, i.e. the propagators for the Fronsdal fields supplemented with derivatives thereof as to obey the free equations (3.7). In some form the unfolded propagators were found in [27]. In [32] it was observed that the propagators are simple functions that depend on a few universal geometrical data, which is the spinorial analog of [51]. 


\subsection{Definitions}

One of the basic objects is the Witten bulk-to-boundary propagator for the scalar field from the bulk point $x^{\underline{m}}=\left(\mathrm{x}^{i}, z\right)$ to the boundary point $\mathrm{x}_{a}$

$$
K_{a}=\frac{z}{\left(\mathrm{x}-\mathrm{x}_{a}\right)^{2}+z^{2}}
$$

We often set $\mathrm{x}_{a}=0$ in this section as the generic point can be recovered thanks to the threedimensional Poincaré invariance. The propagator is the boundary limit of the geodesic distance, which the bulk-to-bulk propagator should depend on. It obeys the regular $(\Delta=1)$ boundary condition. The propagator $K$ can be used to define a wave-vector, which turns into bi-spinor $F^{\alpha \dot{\alpha}}$ in the $4 d$ spinorial language:

$$
d \ln K=F_{\alpha \dot{\alpha}} h^{\alpha \dot{\alpha}},
$$

where

$$
F^{\alpha \dot{\alpha}}=\left(\frac{2 z}{\mathrm{x}^{2}+z^{2}} \mathrm{x}^{\alpha \dot{\alpha}}-\frac{\mathrm{x}^{2}-z^{2}}{\mathrm{x}^{2}+z^{2}} i \epsilon^{\alpha \dot{\alpha}}\right)
$$

It will play the same role as the on-shell momentum $p$ plays for $e^{i p x}$ and is the boundary limit of the vector that is tangent to the geodesic connecting two bulk points. There also exist the parallel-transport bi-spinors $\Pi^{\alpha \beta}$ and $\bar{\Pi}^{\dot{\alpha} \beta}$ given by

$$
\Pi^{\alpha \beta}=K\left(\frac{1}{\sqrt{z}} \mathrm{x}^{\alpha \beta}+\sqrt{z} i \epsilon^{\alpha \beta}\right), \quad \bar{\Pi}^{\dot{\alpha} \beta}=K\left(\frac{1}{\sqrt{z}} \mathrm{x}^{\dot{\alpha} \beta}-\sqrt{z} i \epsilon^{\dot{\alpha} \beta}\right)=\left(\Pi^{\alpha \beta}\right)^{\dagger}
$$

that allow one to propagate the boundary polarization spinors into the bulk:

$$
\xi^{\alpha}=\Pi^{\alpha \beta} \eta_{\beta} e^{+i \frac{\pi}{4}}, \quad \bar{\xi}^{\dot{\alpha}}=\left(\xi^{\alpha}\right)^{\dagger}=\bar{\Pi}^{\dot{\alpha} \beta} \eta_{\beta} e^{-i \frac{\pi}{4}} .
$$

This is the full set of the data that any propagator can depend on. The set is closed under covariant derivatives:

$$
\nabla F^{\alpha \dot{\alpha}}=h^{\alpha \dot{\alpha}}+F_{\dot{\gamma}}^{\alpha} h^{\delta \dot{\gamma}} F_{\delta}^{\dot{\alpha}}=0,
$$

and the parallel transported spinors obey

$$
\nabla \xi^{\alpha}-F^{\alpha} \dot{\gamma} \xi_{\delta} h^{\delta \dot{\gamma}}=0, \quad \nabla \xi^{\dot{\alpha}}-F_{\delta}^{\dot{\alpha}} \xi_{\dot{\gamma}} h^{\delta \dot{\gamma}}=0
$$

In practice it is useful to rewrite the Lorentz-covariant derivatives with all indices being explicit:

$$
\nabla_{\alpha \dot{\alpha}} K=K F_{\alpha \dot{\alpha}}, \quad \nabla_{\alpha \dot{\alpha}} \xi_{\beta}=F_{\beta \dot{\alpha}} \xi_{\alpha}, \quad \nabla_{\alpha \dot{\alpha}} \bar{\xi}_{\dot{\beta}}=F_{\alpha \dot{\beta}} \xi_{\dot{\alpha}}, \quad \nabla_{\alpha \dot{\alpha}} F_{\beta \dot{\beta}}=2 \epsilon_{\alpha \beta} \epsilon_{\dot{\alpha} \dot{\beta}}+F_{\alpha \dot{\alpha}} F_{\beta \dot{\beta}}
$$

As a consequence of the differential constraints above one also finds

$$
(\square-4) K=0, \quad(\square-6) F^{\alpha \dot{\alpha}}=0, \quad(\square-4) \xi^{\alpha}=0, \quad(\square-4) \bar{\xi}^{\dot{\alpha}}=0 .
$$




\subsection{Algebraic identities}

The quantities defined above obey several algebraic identities. The wave-vector satisfies

$$
F^{\alpha \dot{\alpha}} F^{\beta}{ }_{\alpha}=\epsilon^{\alpha \beta}, \quad F^{\alpha \dot{\alpha}} F_{\alpha}^{\dot{\beta}}=\epsilon^{\dot{\alpha} \dot{\beta}} .
$$

It behaves in many respects as a 'symplectic' structure that converts the dotted and undotted indices to each other as follows

$$
\begin{aligned}
& F_{\dot{\gamma}}^{\alpha} \bar{\Pi}^{\dot{\gamma} \beta}=i \Pi^{\alpha \beta}, \quad F_{\gamma}^{\dot{\alpha}} \Pi^{\gamma \beta}=-i \bar{\Pi}^{\dot{\alpha} \beta}, \quad \Pi_{\gamma}^{\alpha} \bar{\Pi}^{\dot{\alpha} \gamma}=-i K F^{\alpha \dot{\alpha}}, \\
& \xi^{\alpha}=F_{\dot{\alpha}}^{\alpha} \bar{\xi}^{\dot{\alpha}}, \quad \bar{\xi}^{\dot{\alpha}}=\xi^{\alpha} F_{\alpha}^{\dot{\alpha}} .
\end{aligned}
$$

The parallel-transport bi-spinors $\Pi^{\alpha \beta}$ and $\bar{\Pi}^{\dot{\alpha} \beta}$ obey identities similar to (4.10):

$$
\begin{array}{ll}
\Pi^{\alpha \beta} \Pi_{\beta}^{\gamma}=K \epsilon^{\alpha \gamma}, & \bar{\Pi}^{\dot{\alpha} \beta} \bar{\Pi}_{\beta}^{\dot{\gamma}}=K \epsilon^{\dot{\alpha} \dot{\gamma}}, \\
\Pi^{\beta \alpha} \Pi_{\beta}{ }^{\gamma}=K \epsilon^{\alpha \gamma}, & \bar{\Pi}^{\dot{\beta} \alpha} \bar{\Pi}_{\dot{\beta}}^{\gamma}=K \epsilon^{\alpha \gamma} .
\end{array}
$$

There are also useful identities involving the one-form $h^{\alpha \dot{\alpha}}=d x^{\underline{m}} h_{\underline{m}}^{\alpha \dot{\alpha}}$ :

$$
\begin{aligned}
(F \cdot h) F^{\alpha \dot{\alpha}}+h^{\alpha \dot{\alpha}} & =F_{\dot{\beta}}^{\alpha} h^{\beta \dot{\beta}} F_{\beta}{ }^{\dot{\alpha}}, \\
(F \cdot h) \xi^{\alpha}+\left(F_{\dot{\gamma}}^{\alpha} h_{\beta}{ }^{\dot{\gamma}}-F_{\beta \dot{\gamma}} h^{\alpha \dot{\gamma}}\right) \xi^{\beta} & =0,
\end{aligned}
$$

which result from the fact that anti-symmetrization over any three spinorial indices vanishes identically.

\subsection{Inversion map}

The basic computational tool we will employ is based on the inversion trick [52]. For that reason it is important to know the transformation properties of the variables defined above under the inversion isometry of $A d S_{4}$. Since the boundary-to-bulk objects $K, F, \xi$ and $\bar{\xi}$ depend both on the bulk point, on the boundary point and polarization spinor $\eta$, the inversion map on the AdS-side should be accompanied by the inversion map on the boundary. Using the inversion map rules (2.3), we derive the following transformation properties:

$$
\begin{aligned}
K\left(R(\mathrm{x}, z) ; R \mathrm{x}_{i}\right) & =\mathrm{x}_{i}^{2} K\left(\mathrm{x}, z ; \mathrm{x}_{i}\right), \\
\Pi^{\alpha \beta}\left(R(\mathrm{x}, z) ; R\left(\mathrm{x}_{i}\right)\right) & =-J^{\alpha} \dot{\gamma}^{\dot{\gamma}}{ }_{\delta}\left(\mathrm{x}, z ; \mathrm{x}_{i}\right) \mathrm{x}_{i}^{\delta \beta} \\
\xi^{\alpha}\left(R(\mathrm{x}, z) ; R\left(\mathrm{x}_{i}, \eta_{i}\right)\right) & =+i J_{\dot{\gamma}}^{\alpha} \bar{\xi}^{\dot{\gamma}}\left(\mathrm{x}, z ; \mathrm{x}_{i}, \eta_{i}\right), \\
\bar{\xi}^{\dot{\alpha}}\left(R(\mathrm{x}, z) ; R\left(\mathrm{x}_{i}, \eta_{i}\right)\right) & =-i J_{\gamma}{ }^{\dot{\alpha}} \xi^{\gamma}\left(\mathrm{x}, z ; \mathrm{x}_{i}, \eta_{i}\right), \\
F^{\alpha \dot{\alpha}}\left(R(\mathrm{x}, z) ; R\left(\mathrm{x}_{i}\right)\right) & =J_{\dot{\beta}}^{\alpha} J_{\beta}{ }^{\dot{\alpha}} F^{\beta \dot{\beta}}\left(\mathrm{x}, z ; \mathrm{x}_{i}\right),
\end{aligned}
$$

where we defined $J^{\alpha \dot{\alpha}}$ as

$$
J^{\alpha \dot{\alpha}}=\frac{x^{\alpha \dot{\alpha}}}{\sqrt{x^{2}}}=\frac{\mathrm{x}^{\alpha \dot{\alpha}}+i z \epsilon^{\alpha \dot{\alpha}}}{\sqrt{\mathrm{x}^{2}+z^{2}}}, \quad \quad J_{\dot{\gamma}}^{\alpha} J^{\beta \dot{\gamma}}=-\epsilon^{\alpha \beta} .
$$

\section{$5 \quad$ Vertices and propagators}

In this section we discuss the boundary-to-bulk propagators for HS fields and evaluate the vertex (3.16) on the propagators. 


\subsection{Propagators}

With the help of the geometric objects introduced in section 4 it is very easy to construct propagators. First of all, there is a unique expression for the Fronsdal field propagator:

$$
\Phi_{\alpha(s), \dot{\alpha}(s)}=-2 \sigma^{2 s} A \frac{\Gamma[s] \Gamma[s]}{\Gamma[2 s]}\left[K \xi_{\alpha(s)} \bar{\xi}_{\dot{\alpha}(s)}\right],
$$

where $A$ is the factor from the free unfolded equations (3.11), $\sigma$ is a parameter that counts spin, the $\Gamma$-functions will be explained later as the most convenient normalization.

In practice we need the propagators for $\omega$ and $C$ fields that enter the unfolded equations. These fields encode derivatives of the Fronsdal field. The propagators can be written in a very compact form as ${ }^{15}$

$$
\begin{aligned}
\omega & =-2 \sigma^{2} A K h_{\alpha \dot{\alpha}} \xi^{\alpha} \bar{\xi}^{\dot{\alpha}} \int_{0}^{1} d t \exp i\left[\sigma t y^{\alpha} \xi_{\alpha}-(1-t) \sigma \bar{y}^{\dot{\alpha}} \bar{\xi}_{\dot{\alpha}}\right], \\
C & =K \exp i\left[-y_{\alpha} F^{\alpha \dot{\alpha}} \bar{y}_{\dot{\alpha}}+\sigma y^{\alpha} \xi_{\alpha}+\theta\right]+K \exp i\left[-y_{\alpha} F^{\alpha \dot{\alpha}} \bar{y}_{\dot{\alpha}}-\sigma \bar{y}^{\dot{\alpha}} \bar{\xi}_{\alpha}-\theta\right], \\
& =K \exp i\left[-y_{\alpha} F^{\alpha \dot{\alpha}} \bar{y}_{\dot{\alpha}}+\sigma y^{\alpha} \xi_{\alpha}+\theta\right]+h . c .,
\end{aligned}
$$

where $A$ is a constant that is related to the normalization of the free unfolded equations (3.11). The h.c. operation is defined as

$$
\text { h.c. }(\xi)=-\bar{\xi}, \quad \text { h.c. }(\theta)=-\theta
$$

and $\sigma$ is just a factor that counts spins, so can be put to one or any $\sigma(s)$. We will fix this normalization later.

\subsection{Vertices}

Let us remind that the equation we will extract the correlation function from is

$$
\widetilde{\mathrm{D}} C^{(2)}=\omega \star C-C \star \pi(\omega)+\mathcal{O}\left(C^{2}\right),
$$

where we evaluate the r.h.s. on the propagators and then solve for the Klein-Gordon equation. Expanding the master zero-form $C$ as

$$
C(y, \bar{y} \mid x)=\phi(x)+\phi_{\alpha \dot{\alpha}}(x) y^{\alpha} \bar{y}^{\dot{\alpha}}+\cdots
$$

the Klein-Gordon equation with a source is hidden in the first order form:

$$
\begin{aligned}
d \phi-i h_{\alpha \dot{\alpha}} \phi^{\alpha \dot{\alpha}} & =h^{\alpha \dot{\alpha}} P_{\alpha \dot{\alpha}}, \\
\nabla \phi^{\alpha \dot{\alpha}}+h^{\alpha \dot{\alpha}} \phi+\ldots & =h^{\beta \dot{\beta}} P_{\beta \dot{\beta}}^{\alpha \dot{\alpha}},
\end{aligned}
$$

\footnotetext{
${ }^{15}$ The unfolded propagators were first found in [27] in a different form, especially the $\omega$ propagator where the gauge ambiguity is essential. The $C$ propagator was cast into the form below in [32], see also [29, 31, 40]. The simple expression for the $\omega$ propagator was obtained together with Slava Didenko and was not published by us before.
} 
where the one-form $\mathcal{P}=h^{\alpha \dot{\alpha}} P_{\alpha \dot{\alpha}}$ denotes the source built out of the free fields:

$$
\mathcal{P}=\omega \star C-C \star \pi(\omega)
$$

and we need the first two Taylor coefficients only:

$$
\mathcal{P}=h^{\alpha \dot{\alpha}} P_{\alpha \dot{\alpha}}(y, \bar{y})=h^{\alpha \dot{\alpha}}\left[P_{\alpha \dot{\alpha}}+P_{\alpha \dot{\alpha}}^{\beta \dot{\beta}} y_{\beta} \bar{y}_{\dot{\beta}}+\cdots\right] .
$$

The first constraint can be solved for the auxiliary field $\phi^{\alpha \dot{\alpha}}$ and the result then plugged into the second one to get the Klein-Gordon equation with a source [3]:

$$
(\square-4) \phi=\left.\left[\nabla^{\alpha \dot{\alpha}} P_{\alpha \dot{\alpha}}+i \partial^{\alpha} \partial^{\dot{\alpha}} P_{\alpha \dot{\alpha}}\right]\right|_{y, \bar{y}=0} .
$$

Since the propagators are known, we can get the source explicitly as

$$
\begin{aligned}
(\square-4) \phi & =-4 A K_{1} K_{2} \int_{0}^{1} d t\left[(1+h . c .)\left(1+\pi_{\xi_{1}}\right)\left(w+i t(1-t) w^{2}+i t w \xi_{1} \xi_{2}\right) B\right] \\
B & =\exp i\left[t(1-t) w+t \xi_{1} \xi_{2}+\theta\right] \\
w & =\left(\xi_{1} F_{2} \bar{\xi}_{1}\right)
\end{aligned}
$$

where $K_{1}, \xi_{1}$ refer to $\omega$ and $K_{2}, \xi_{2}, F_{2}$ to $C$, and

$$
\left(\xi_{1} \xi_{2}\right) \equiv \xi_{1}^{\alpha} \xi_{2 \alpha}, \quad\left(\xi_{1} F_{2} \bar{\xi}_{1}\right) \equiv \xi_{1 \alpha} F^{\alpha \dot{\alpha}} \xi_{1 \dot{\alpha}}
$$

Here $\pi_{\xi}$ is the twist map that is now realized on $\xi$ (or $\bar{\xi}$ due to the bosonic projection), $\pi(\omega)=\omega(-\xi, \bar{\xi})=\omega(\xi,-\bar{\xi})$. The appearance of $\pi_{\xi_{1}}$ is responsible for the difference between HS fields with and without additional Yang-Mills groups. If we keep $\pi_{\xi_{1}}$ then in the dual CFT the correlators $\left\langle j_{s_{1}} j_{s_{2}} j_{0}\right\rangle$ will vanish for $s_{1}+s_{2}$ odd. If we drop $\pi_{\xi_{1}}$ then on the CFT side $\left\langle j_{s_{1}} j_{s_{2}} j_{0}\right\rangle$ does not vanish for $s_{1}+s_{2}$ odd and therefore we have a leftover global symmetry group. The latter case is more general and is easier to deal with.

On expanding the generating function (5.10b) and picking the terms of spins $s_{1}$ from $\omega$ and $s_{2}$ from $C$ we find the vertex evaluated on the propagators in a very simple form ${ }^{16}$

$$
\begin{aligned}
(\square-4) \phi & =-4 A K_{1} K_{2} \sum_{s_{1}, s_{2}} V_{s_{1}, s_{2}, 0}, \\
V_{s_{1}, s_{2}, 0} & =v_{s_{1}, s_{2}, 0}\left[\left(\xi_{1} F_{2} \bar{\xi}_{1}\right)^{s_{1}-s_{2}}\left(\xi_{1} \xi_{2}\right)^{2 s_{2}} e^{i \theta}+h . c .\right] \\
v_{s_{1}, s_{2}, 0} & =\frac{i^{s_{1}+s_{2}-1} \Gamma\left(s_{1}+s_{2}+1\right)}{\Gamma\left(2 s_{1}\right) \Gamma\left(2 s_{2}+1\right)},
\end{aligned}
$$

for $s_{1}>s_{2}$. As we already commented in the Introduction, the vertex above can be used to obtain $0-s_{1}-s_{2}$ correlators for $s_{1} \neq s_{2}$, but extrapolation to $s_{1}=s_{2}$ will give the correct answer too since the correlation function depends smoothly on spins. Nevertheless, the $0-s-s$ correlators should originate from the $C C$-terms in $(3.13 \mathrm{~b})$, which previously

\footnotetext{
${ }^{16}$ This vertex is also present in [27], but it does not seem to have such a simple form as below, which should be related to the $\omega$ propagator being in a different gauge.
} 
gave infinite result $[27,53]$. Therefore, we would like to use the proposal of [34], where the new $C C$-terms are

$$
\begin{aligned}
\mathcal{U}(h, C, C)= & \frac{1}{4} \int_{0}^{1} d \tau \int \frac{d \bar{u} d \bar{v}}{(2 \pi)^{2}} e^{i \bar{u}_{\dot{\beta}} \bar{v}^{\dot{\beta}}} h_{\alpha \dot{\alpha}} y^{\alpha}[\tau \bar{u}+(1-\tau) \bar{v}]^{\dot{\alpha}} C(\tau y, \bar{y}+\bar{u}) C((1-\tau) y, \bar{y}+\bar{v}) \\
& + \text { h.c. }
\end{aligned}
$$

A simple computation along the lines above ${ }^{17}$ gives $^{18}$

$$
(\square-4) \phi=-4 A K_{1} K_{2} \sum_{s} V_{s, s, 0} .
$$

Therefore, the $0-s-s$ vertex turns out to have the same form as the naive extrapolation of (5.12) and will give the correct answer without any additional computation needed once (5.12) is shown to be correct.

\section{Computation of the cubic amplitude}

From the bulk vertex (5.12b), the Witten diagram amplitude for $\left\langle J_{s_{1}} J_{s_{2}} J_{0}\right\rangle$ for $s_{1}>s_{2}$ is obtained as

$$
\begin{aligned}
& \left\langle J_{s_{1}}\left(\mathrm{x}_{1}, \eta_{1}\right) J_{s_{2}}\left(\mathrm{x}_{2}, \eta_{2}\right) J_{0}\left(\mathrm{x}_{3}\right)\right\rangle_{h . s .}=(-4 A) c_{s_{1}} c_{s_{2}} c_{0} v_{s_{1}, s_{2}, 0} \times \\
& \times \int \frac{d^{3} \mathrm{x} d z}{z^{4}} K_{1} K_{2}\left(K_{3}\right)^{\Delta}\left(\xi_{1} F_{2} \bar{\xi}_{1}\right)^{s_{1}-s_{2}}\left[\left(\xi_{1} \xi_{2}\right)^{2 s_{2}} e^{i \theta}+\left(\bar{\xi}_{1} \bar{\xi}_{2}\right)^{2 s_{2}} e^{-i \theta}\right],
\end{aligned}
$$

where

$K_{i}=K\left(\mathrm{x}-\mathrm{x}_{i}, z\right), \quad F_{i}^{\alpha \dot{\alpha}}=F^{\alpha \dot{\alpha}}\left(\mathrm{x}-\mathrm{x}_{i}, z\right), \quad \xi_{i}^{\alpha}=\xi^{\alpha}\left(\mathrm{x}-\mathrm{x}_{i}, z ; \eta_{i}\right), \quad \bar{\xi}_{i}^{\dot{\alpha}}=\bar{\xi}^{\dot{\alpha}}\left(\mathrm{x}-\mathrm{x}_{i}, z ; \eta_{i}\right)$.

The factor $(-4 A)$ in (6.1) does not have any physical meaning and is an arbitrary normalization factor between $\omega$ and $C$ in (3.11). For convenience, we reproduce the factor

$$
v_{s_{1}, s_{2}, 0}=\frac{i^{s_{1}+s_{2}-1} \Gamma\left(s_{1}+s_{2}+1\right)}{\Gamma\left(2 s_{1}\right) \Gamma\left(2 s_{2}+1\right)} .
$$

Also, we introduced normalization factors $c_{s}$ for each of the three fields. These cannot be fixed from the equations of motion and correspond to a freedom on the CFT side to normalize at will the two-point functions $\left\langle j_{s} j_{s}\right\rangle$. Lastly, there are two options for boundary conditions on the scalar fields: $\Delta=1$ and $\Delta=2$.

The three-point integrals are doable in principle due to the fact that one can always 'scalarize' the integrand by representing all $x^{\alpha \dot{\alpha}}$-factors as derivatives with respect to the boundary points $\mathrm{x}_{i}$. The scalar three-point integral was done long ago in [52]. The problem is to scalarize in the most efficient way as to break as less symmetries as possible. We extend

\footnotetext{
${ }^{17}$ E.Sk is grateful to Massimo Taronna with whom this was obtained last June, see also [68].

${ }^{18}$ The issue of $\theta$-dependence is unclear to us since in [34] it was proposed to take the $\mathcal{N}=2$ supersymmetric HS model and truncate it to the bosonic one using the boundary conditions that contain $\theta$-dependence. Nevertheless, our bosonic truncation which does not rely on imposition of $\theta$-dependent boundary conditions gives the right answer.
} 
the inversion method of [52] to our case. Firstly, using the translation invariance we can set $\mathrm{x}_{1}=0$. Then, we apply the inversion map both to the boundary and bulk data. As a result the basic structures that enter the integrand drastically simplify:

$$
\begin{aligned}
\frac{d^{3} \mathrm{x} d z}{z^{4}} & \rightarrow \frac{d^{3} \mathrm{x} d z}{z^{4}}, \\
K_{1} & \rightarrow z, \\
K_{2,3} & \rightarrow \mathrm{x}_{2,3}^{2} K_{2,3}, \\
\left(\xi_{1} F_{2} \bar{\xi}_{1}\right) & \rightarrow 2 z K_{2}\left[\eta_{1}\left(\mathrm{x}-\mathrm{x}_{2}\right) \eta_{1}\right]=-2 z K_{2} T_{11}, \\
\left(\xi_{2} F_{1} \bar{\xi}_{2}\right) & \rightarrow 2 K_{2}^{2}\left[\eta_{2}\left(\mathrm{x}-\mathrm{x}_{2}\right) \eta_{2}\right]=-2 K_{2}^{2} T_{22}, \\
\left(\xi_{1} \xi_{2}\right)+\left(\bar{\xi}_{1} \bar{\xi}_{2}\right) & \rightarrow-2 z K_{2}\left(\eta_{1} \eta_{2}\right), \\
\left(\xi_{1} \xi_{2}\right)-\left(\bar{\xi}_{1} \bar{\xi}_{2}\right) & \rightarrow 2 i K_{2}\left[\eta_{1}\left(\mathrm{x}-\mathrm{x}_{2}\right) \eta_{2}\right]=-2 i K_{2} T_{12},
\end{aligned}
$$

where we defined

$$
T_{i j}=-\left[\eta_{\alpha}^{i}\left(\mathrm{x}-\mathrm{x}_{2}\right)^{\alpha \beta} \eta_{\beta}^{j}\right],
$$

and we will use the same notation for the variables after the inversion is applied. Our strategy is to rewrite the integrand in terms of simple differential operators acting on a scalar integrand

$$
\int \frac{d^{3} \mathrm{x} d z}{z^{4}} z^{a}\left(K_{2}\right)^{b}\left(K_{3}\right)^{\Delta}=\left(\mathrm{x}_{23}\right)^{a-b-\Delta} I_{a, b, \Delta}
$$

where

$$
I_{a, b, \Delta}=\frac{\pi^{3 / 2} \Gamma\left(\frac{1}{2}(a+b-\Delta)\right) \Gamma\left(\frac{1}{2}(a-b+\Delta)\right) \Gamma\left(\frac{1}{2}(-a+b+\Delta)\right) \Gamma\left(\frac{1}{2}(a+b+\Delta-3)\right)}{2 \Gamma(a) \Gamma(b) \Gamma(\Delta)} .
$$

There are three operators that can be immediately observed to generate the same structures that occur under the integral sign:

$$
O_{11}=\left(\eta_{1} \partial_{2} \eta_{1}\right) \equiv \eta_{1}^{\alpha} \frac{\partial}{\partial \mathrm{x}_{2}^{\alpha \beta}} \eta_{1}^{\beta}, \quad O_{12}=\left(\eta_{1} \partial_{2} \eta_{2}\right), \quad O_{22}=\left(\eta_{2} \partial_{2} \eta_{2}\right)
$$

The operators act on $K_{2}$ factors only and yield:

$$
O_{i j} f\left(K_{2}\right)=\frac{\left(K_{2}\right)^{2}}{z} \frac{\partial}{\partial K_{2}} f\left(K_{2}\right) T_{i j}
$$

There is one relation between $T_{i j}$ that is the bulk analog of the $S_{3}^{2}+Q_{1} Q_{2}-P_{12}^{2} \equiv 0$ relation:

$$
\left(T_{12}\right)^{2}=T_{11} T_{22}+\mathrm{x}_{23}^{2}\left(\eta_{1} \eta_{2}\right)^{2}
$$

The integrand can be represented as a function of $O_{i j}$ and $\left(\eta_{1} \eta_{2}\right)$ acting on the scalar integrand, and there is more than one way to do so due to the identity above. Then, the integral can be done and one is left with the same functional acting on some powers of $\left|\mathrm{x}_{23}\right|$, which clearly generates some function of the conformally invariant structures resulting from 
setting $\mathrm{x}_{1}=0$ followed by the inversion map:

$$
\begin{aligned}
\mathrm{x}_{2,3} & \rightarrow \frac{1}{\mathrm{x}_{2,3}}, \\
\mathrm{x}_{23} & \rightarrow \frac{\mathrm{x}_{23}}{\mathrm{x}_{2} \mathrm{x}_{3}}, \\
P_{12} & \rightarrow\left(\eta_{1} \eta_{2}\right), \\
Q_{1} & \rightarrow-\left[\eta_{1} \mathrm{x}_{23} \eta_{1}\right], \\
Q_{2} & \rightarrow+\left[\eta_{2} \mathrm{x}_{23} \eta_{2}\right] \frac{1}{\mathrm{x}_{23}^{2}}, \\
S_{3} & \rightarrow+\left[\eta_{1} \mathrm{x}_{23} \eta_{2}\right] \frac{1}{\mathrm{x}_{23}} .
\end{aligned}
$$

It is convenient to use the same notation $T_{i j}$ for the corresponding structures on the boundary $T_{i j}=\left[\eta_{i} \mathrm{x}_{23} \eta_{j}\right]$ since they arise under the action of $O_{i j}$ on $\mathrm{x}_{23}$ resulting from the integral. In other words, if $O_{i j}$ applied to the l.h.s. (6.6), it generates bulk $T_{i j}$ defined in (6.5), and if $O_{i j}$ acts on the r.h.s. of (6.6), it generates the boundary $T_{i j}$.

\subsection{Leading coefficients}

Our goal is to reproduce the full structure of the three-point functions. However, it is useful to perform a few simple checks of the duality that do not require establishing a full dictionary between bulk and boundary. It is clear that $O_{i j}$ operators when applied one after another produce

$$
O_{11}^{n_{1}} O_{12}^{n_{2}} O_{22}^{n_{3}}\left(K_{2}\right)^{a}=\frac{\Gamma[a+n]}{z^{n} \Gamma[a]}\left(K_{2}\right)^{a+n} T_{11}^{n_{1}} T_{12}^{n_{2}} T_{22}^{n_{3}}+\mathcal{O}\left(\eta_{1} \eta_{2}\right),
$$

where $n=n_{1}+n_{2}+n_{3}$. Therefore, up to $P_{12}$-terms, which are represented by $\mathcal{O}\left(\eta_{1} \eta_{2}\right)$ after the inversion, the bulk computation amounts to pulling out the $T_{i j}$ structures as powers of $O_{i j}$, computing the scalar integral and then pushing the $O_{i j}$ factors in. In the last step operators $O_{i j}$ act on $\mathrm{x}_{23}$ resulting from the bulk integral and generate $Q_{1,2}$ modulo $Q_{1} Q_{2} \sim-S_{3}^{2}$.

Type-A, $\boldsymbol{\Delta}=\mathbf{1}$. In the case of $\Delta=1$ and $\theta=0$ a simple computation gives:

$$
\left\langle J_{s_{1}}\left(\mathrm{x}_{1}, \eta_{1}\right) J_{s_{2}}\left(\mathrm{x}_{2}, \eta_{2}\right) J_{0}\left(\mathrm{x}_{3}\right)\right\rangle_{h . s .}=(-4 A) c_{s_{1}} c_{s_{2}} c_{0} v_{s_{1}, s_{2}, 0} I_{s_{1}, s_{2}, 0}^{\Delta=1} \times\left[\left(Q_{1}\right)^{s_{1}}\left(Q_{2}\right)^{s_{2}}+\mathcal{O}\left(P_{12}\right)\right],
$$

where $I_{s_{1}, s_{2}, 0}$ is the factor that comes from the bulk

$$
I_{s_{1}, s_{2}, 0}^{\Delta=1}=\frac{\pi^{3}\left(-\frac{1}{2}\right)^{s_{1}+s_{2}}(-)^{s_{1}} \Gamma\left(s_{1}+\frac{1}{2}\right)}{s_{1} \Gamma\left(\frac{1}{2}-s_{2}\right) \Gamma\left(s_{1}+s_{2}+1\right)} .
$$

This should be compared with the generating function (2.18) in free boson theory: ${ }^{19}$

$$
\left\langle J_{s_{1}}\left(\mathrm{x}_{1}, \eta_{1}\right) J_{s_{2}}\left(\mathrm{x}_{2}, \eta_{2}\right) J_{0}\left(\mathrm{x}_{3}\right)\right\rangle_{f . b .}=\frac{2}{\mathrm{x}_{12} \mathrm{x}_{23} \mathrm{x}_{13}}\left(\frac{i}{2}\right)^{s_{1}+s_{2}} \frac{1}{s_{1} ! s_{2} !}\left[\left(Q_{1}\right)^{s_{1}}\left(Q_{2}\right)^{s_{2}}+\mathcal{O}\left(P_{12}\right)\right]
$$

\footnotetext{
${ }^{19}$ What we compute would correspond to the dual theory with $\mathrm{U}(M)$ global symmetry $(2.20)$. The same comment applies to all correlators below.
} 
Since the normalization of the boundary-to-bulk propagators is not yet fixed, we should compare two function of $s_{1}, s_{2}$, the bulk result and the CFT result, up to a product of functions of $s_{1}$ and $s_{2}$ separately. In doing so we can find that the normalization factor is

$$
c_{s}=-\frac{4^{s}}{\pi} .
$$

With this normalization we have a perfect match up to the terms of order $\left(\eta_{1} \eta_{2}\right)=P_{12}$, namely

$$
\left\langle J_{s_{1}}\left(\mathrm{x}_{1}, \eta_{1}\right) J_{s_{2}}\left(\mathrm{x}_{2}, \eta_{2}\right) J_{0}\left(\mathrm{x}_{3}\right)\right\rangle_{h . s .}=\left\langle J_{s_{1}}\left(\mathrm{x}_{1}, \eta_{1}\right) J_{s_{2}}\left(\mathrm{x}_{2}, \eta_{2}\right) J_{0}\left(\mathrm{x}_{3}\right)\right\rangle_{f . b .}+\mathcal{O}\left(P_{12}\right) .
$$

Type-B, $\Delta=2$. The same computation but for $\Delta=2$ and $\theta=\pi / 2$ leads to

$$
\begin{aligned}
\left\langle J_{s_{1}}\left(\mathrm{x}_{1}, \eta_{1}\right) J_{s_{2}}\left(\mathrm{x}_{2}, \eta_{2}\right) J_{0}\left(\mathrm{x}_{3}\right)\right\rangle_{h . s .}= & (-4 A) c_{s_{1}} c_{s_{2}} \tilde{c}_{0} v_{s_{1}, s_{2}, 0} I_{s_{1}, s_{2}, 0}^{\Delta=2} \\
& \times\left[\left(Q_{1}\right)^{s_{1}-1}\left(Q_{2}\right)^{s_{2}-1} P_{12} S_{3}+\mathcal{O}\left(P_{12}^{2}\right)\right] .
\end{aligned}
$$

Here we assumed that the normalization of the scalar field propagator $\tilde{c}_{0}$ can be different from $c_{0}$ due to the change in boundary conditions. The bulk integral and other factors combine into $I_{s_{1}, s_{2}, 0}^{\Delta=2}$ as follows

$$
I_{s_{1}, s_{2}, 0}^{\Delta=2}=-\frac{\pi^{3} s_{2}\left(-\frac{1}{2}\right)^{s_{1}+s_{2}-1}(-)^{s_{1}} \Gamma\left(s_{1}+\frac{1}{2}\right)}{\Gamma\left(\frac{1}{2}-s_{2}\right) \Gamma\left(s_{1}+s_{2}+1\right)} .
$$

We note that one factor of $P_{12}$ jumps out of the integral and the subleading terms are of order $P_{12}^{2}$. The result should be compared with the generating function (2.28) in free fermion theory:

$$
\begin{aligned}
\left\langle J_{s_{1}}\left(\mathrm{x}_{1}, \eta_{1}\right) J_{s_{2}}\left(\mathrm{x}_{2}, \eta_{2}\right) J_{0}\left(\mathrm{x}_{3}\right)\right\rangle_{f . f .}= & \frac{i^{s_{1}+s_{2}-2}}{\mathrm{x}_{23}^{2} \mathrm{x}_{13}^{2} 2^{s_{1}+s_{2}-2}} \frac{2}{\left(s_{1}-1\right) !\left(s_{2}-1\right) !} \\
& \times\left[\left(Q_{1}\right)^{s_{1}-1}\left(Q_{2}\right)^{s_{2}-1} S_{3} P_{12}+\mathcal{O}\left(P_{12}^{2}\right)\right] .
\end{aligned}
$$

We find the two results to agree, namely

$$
\left\langle J_{s_{1}}\left(\mathrm{x}_{1}, \eta_{1}\right) J_{s_{2}}\left(\mathrm{x}_{2}, \eta_{2}\right) J_{0}\left(\mathrm{x}_{3}\right)\right\rangle_{h . s .}=-\frac{\tilde{c}_{0}}{c_{0}}\left\langle J_{s_{1}}\left(\mathrm{x}_{1}, \eta_{1}\right) J_{s_{2}}\left(\mathrm{x}_{2}, \eta_{2}\right) J_{0}\left(\mathrm{x}_{3}\right)\right\rangle_{f . f .}+\mathcal{O}\left(P_{12}\right) .
$$

Once the normalization factors $c_{s}$ are fixed by the Type-A duality the match just observed is even more nontrivial because the only freedom that we have is an overall spin-independent factor. $^{20}$ The canonical normalization of the scalar field propagators [52] is such that $\tilde{c}_{0} / c_{0}=-2$.

\footnotetext{
${ }^{20}$ The generating functions of the HS currents built out of free bosons and fermions, which were introduced in section 2, are components of the supermultiplet of HS currents. Therefore, the normalization of the HS currents in free boson and free fermion are naturally related to each other.
} 


\subsection{Complete dictionary}

We would like to reproduce the full structure of CFT correlators. The idea is to take the subleading terms into account and express the bulk integrand as the action of a differential operator in $O_{i j}$ on the scalar integrand. The operators $O_{i j}$ produce $T_{i j}$ and the subleading terms are obtained due to

$$
O_{11} T_{22}=\left(\eta_{1} \eta_{2}\right)^{2}, \quad O_{22} T_{11}=\left(\eta_{1} \eta_{2}\right)^{2}, \quad O_{12} T_{12}=-\frac{1}{2}\left(\eta_{1} \eta_{2}\right)^{2} .
$$

Operators $O_{i j}$ commute with each other. Moreover, $O_{11}$ does not produce any subleading terms at all. Then the action of any power of, say $O_{12}$, can be evaluated starting from $\left(\mathrm{x} \equiv \mathrm{x}_{23}\right)$

$$
O_{12} f\left(\mathrm{x}^{2}, T_{12}\right)=\left(-T_{12} \frac{\partial}{\partial \mathrm{x}^{2}}-\frac{1}{2}\left(\eta_{1} \eta_{2}\right)^{2} \frac{\partial}{\partial T_{12}}\right) f\left(\mathrm{x}^{2}, T_{12}\right)
$$

and exponentiating it as

$$
\exp \left[t O_{12}\right]\left(\mathrm{x}^{2}\right)^{-a}=\left(\mathrm{x}^{2}-t T_{12}+\frac{t^{2}}{4}\left(\eta_{1} \eta_{2}\right)^{2}\right)^{-a}
$$

which leads to Gegenbauer polynomials:

$$
\left(O_{12}\right)^{n}\left(\mathrm{x}^{2}\right)^{-a}=\sum_{k} A_{k}^{a, n}\left(\eta_{1} \eta_{2}\right)^{2 k}\left(T_{12}\right)^{n-2 k}\left(\mathrm{x}^{2}\right)^{-(a+n-k)}, \quad A_{k}^{a, n}=\frac{(-)^{k} n ! \Gamma(a-k+n)}{4^{k} k ! \Gamma(a)(n-2 k) !} .
$$

In particular, we find (6.12)

$$
\left(O_{12}\right)^{n}\left(\mathrm{x}^{2}\right)^{-a}=\frac{\Gamma[a+n]}{\Gamma[a]}\left(\mathrm{x}^{2}\right)^{-(a+n)}\left(T_{12}\right)^{n}+\mathcal{O}\left(\eta_{1} \eta_{2}\right) .
$$

In fact, we will use the inversion formula:

$$
\sum_{k=0} B_{k}^{a, n}\left(\eta_{1} \eta_{2}\right)^{2 k}\left(O_{12}\right)^{n-2 k}\left(\mathrm{x}^{2}\right)^{-(a+k-n)}=\left(\mathrm{x}^{2}\right)^{-a}\left(T_{12}\right)^{n}, B_{k}^{a, n}=\frac{\Gamma[a+k-n] n !}{\Gamma[a] 4^{k} k !(n-2 k) !}\left(\eta_{1} \eta_{2}\right)^{2 k} .
$$

The same formula works in the bulk if we replace $\left|\mathrm{x}^{2}\right|^{-1}$ by $K_{2} / z$ and $T_{i j}$ by the bulk $T_{i j}$ (6.5) which we happen to denote by the same symbol, as explained below (6.11). Therefore, any $T_{12}$ structure can also be factored out.

\subsection{Complete three-point functions}

We can now compute (6.1) for general $\theta$. The choice $\Delta=1$ should be compared with CS-boson and $\Delta=2$ with CS-fermion.

The computation is now reduced to the following simple steps: (i) express the integrand in terms of $\left(\xi_{1} \xi_{2}\right) \pm\left(\bar{\xi}_{1} \bar{\xi}_{2}\right)$; (ii) apply the inversion map and express the integrand in terms of $T_{i j}$; (iii) $T_{11}$ is factored out immediately, while $T_{12}$ is pull out with the help of (6.27) as certain polynomial in $O_{12}$; (iv) the integral can be done; (v) the operators $O_{i j}$ should be evaluated in terms of $T_{i j}$, which is easy for $O_{11}$ and we use (6.25) for $O_{12}$; (vi) powers of $T_{i j}$ should be replaced by $Q_{1}, Q_{2}, P_{12}, S_{3}$. 
As the first step we need the following simple identity, which reveals the dependence on $\theta$ of the final result as well as how it splits into parity-even and parity-odd structures:

$$
\begin{gathered}
{\left[\left(\xi_{1} \xi_{2}\right)^{2 s_{2}} e^{i \theta}+\left(\bar{\xi}_{1} \bar{\xi}_{2}\right)^{2 s_{2}} e^{-i \theta}\right]=\sum_{k} \frac{2 \cos \theta}{4^{s_{2}}} C_{2 k}^{2 s_{2}}\left[\left(\xi_{1} \xi_{2}\right)+\left(\bar{\xi}_{1} \bar{\xi}_{2}\right)\right]^{2 s_{2}-2 k}\left[\left(\xi_{1} \xi_{2}\right)-\left(\bar{\xi}_{1} \bar{\xi}_{2}\right)\right]^{2 k}+} \\
+\sum_{k} \frac{2 i \sin \theta}{4^{s_{2}}} C_{2 k+1}^{2 s_{2}}\left[\left(\xi_{1} \xi_{2}\right)+\left(\bar{\xi}_{1} \bar{\xi}_{2}\right)\right]^{2 s_{2}-2 k-1}\left[\left(\xi_{1} \xi_{2}\right)-\left(\bar{\xi}_{1} \bar{\xi}_{2}\right)\right]^{2 k+1}
\end{gathered}
$$

Since the $\theta$-dependence is fixed, in computing the correlation functions it is sufficient to choose either $\theta=0$ or $\theta=\pi / 2$ and for each of these cases to consider the $\Delta=1$ and $\Delta=2$ boundary conditions for the scalar field. Therefore, we need to compute four bulk integrals. In fact, these terms can be computed in the parity even Type-A,B theories but with different choice of boundary conditions for the scalar field, see also the end of section 2.6.

The comment that applies to all the cases considered below is that the generating functions of correlators depend smoothly on spins and therefore it should be possible to extrapolate the result in the case of $s_{1}>s_{2}$ to the case of $s_{1}=s_{2}$, which is an argument used in [27]. The assumption $s_{1}>s_{2}$ was used to pick only $\omega_{s_{1}} C_{s_{2}}$ terms and for the opposite situation we find contribution from $\omega_{s_{2}} C_{s_{1}}$, which is the same. Therefore, the computation below covers all possible $s_{1}$ and $s_{2}$.

Another comment is that the results we obtain in the bulk are valid for the case of $\mathrm{U}(N)$ CFT's that have HS currents with all integer spins (there are only HS currents with even spin in the $O(N)$ case). Also, the bulk results are valid for the case of the leftover global symmetry on the CFT side. The projection onto the singlet sector is trivial and can be obtained by taking the bose symmetric part of the correlator, as discussed in section 5.2. It should be noted that the result of [25], which we will compare the AdS/CFT correlators with, were obtained assuming that the prediction of the slightly broken HS symmetry [11] extends to all integers spins and possibly to the case of leftover global symmetries.

\subsubsection{Type-A, free boson}

The integral corresponding to $\Delta=1$ and $\theta=0$ is given by

$$
\begin{aligned}
& \left\langle J_{s_{1}}\left(\mathrm{x}_{1}, \eta_{1}\right) J_{s_{2}}\left(\mathrm{x}_{2}, \eta_{2}\right) J_{0}\left(\mathrm{x}_{3}\right)\right\rangle_{h . s .}^{\Delta=1, \theta=0}=(-4 A) c_{s_{1}} c_{s_{2}} c_{0} v_{s_{1}, s_{2}, 0} \times \\
& \quad \times \int \frac{d^{3} \mathrm{x} d z}{z^{4}} K_{1} K_{2} K_{3}\left(\xi_{1} F_{2} \bar{\xi}_{1}\right)^{s_{1}-s_{2}} \sum_{k} \frac{2}{4^{s_{2}}} C_{2 k}^{2 s_{2}}\left[\left(\xi_{1} \xi_{2}\right)+\left(\bar{\xi}_{1} \bar{\xi}_{2}\right)\right]^{2 s_{2}-2 k}\left[\left(\xi_{1} \xi_{2}\right)-\left(\bar{\xi}_{1} \bar{\xi}_{2}\right)\right]^{2 k} .
\end{aligned}
$$

The final result for the right hand side of this equation is the following triple sum

$$
\begin{aligned}
\sum_{k=0}^{s_{2}} \sum_{i=0}^{k} \sum_{j=0}^{k-i} \frac{(-1)^{k-j} i^{s_{1}+s_{2}} 2^{s_{1}-s_{2}+1} \Gamma\left(2\left(-k+s_{1}+s_{2}\right)\right) \Gamma\left(-i+k+s_{1}-s_{2}+\frac{1}{2}\right)}{\sqrt{\pi} i ! j !\left(2 s_{1}-1\right) !\left(2 s_{2}-2 k\right) !\left(-i+2 s_{1}\right) !(-i-j+k) !} \\
\times \frac{Q_{1}^{s_{1}-s_{2}} P_{12}^{2\left(i+j-k+s_{2}\right)}\left(P_{12}^{2}-Q_{1} Q_{2}\right)^{-i-j+k}}{\mathrm{x}_{12} \mathrm{x}_{13} \mathrm{x}_{23}}
\end{aligned}
$$

where we undid the inversion map. This expression can be supplemented with the $s_{2}>s_{1}$ contribution, extended to diagonal $s_{1}=s_{2}$, as we explained above, then summed over spins 
as to build a generating function, see appendix B.1, giving the result:

$$
\frac{2}{\mathrm{x}_{12} \mathrm{x}_{13} \mathrm{x}_{23}} \exp \left(\frac{i}{2} Q_{1}+\frac{i}{2} Q_{2}\right) \cos P_{12}
$$

This matches exactly the CFT three-point function:

$$
\left\langle J_{s_{1}}\left(\mathrm{x}_{1}, \eta_{1}\right) J_{s_{2}}\left(\mathrm{x}_{2}, \eta_{2}\right) J_{0}\left(\mathrm{x}_{3}\right)\right\rangle_{h . s .}^{\Delta=1, \theta=0}=\left\langle J_{s_{1}}\left(\mathrm{x}_{1}, \eta_{1}\right) J_{s_{2}}\left(\mathrm{x}_{2}, \eta_{2}\right) J_{0}\left(\mathrm{x}_{3}\right)\right\rangle_{f . b .} .
$$

This result is slightly new as we managed to reproduce the full structure of the CFT correlators from the local HS equations where the field theory methods can be applied, and we note that no regularization of infinities is required. The leading coefficients were found in [27] and the same generating function resulted from the non-local $C C$-terms after some regularization in [29].

\subsubsection{Type-B, free fermion}

The integral corresponding to $\Delta=2$ and $\theta=\pi / 2$ reads

$$
\begin{aligned}
& \left\langle J_{s_{1}}\left(\mathrm{x}_{1}, \eta_{1}\right) J_{s_{2}}\left(\mathrm{x}_{2}, \eta_{2}\right) J_{0}\left(\mathrm{x}_{3}\right)\right\rangle_{h . s .}^{\Delta=2, \theta=\frac{\pi}{2}}=(-4 A) c_{s_{1}} c_{s_{2}} \tilde{c}_{0} v_{s_{1}, s_{2}, 0} \times \\
& \times \int \frac{d^{3} \mathrm{x} d z}{z^{4}} K_{1} K_{2} K_{3}^{2}\left(\xi_{1} F_{2} \bar{\xi}_{1}\right)^{s_{1}-s_{2}} \sum_{k} \frac{2 i}{4^{s_{2}}} C_{2 k+1}^{2 s_{2}}\left[\left(\xi_{1} \xi_{2}\right)+\left(\bar{\xi}_{1} \bar{\xi}_{2}\right)\right]^{2 s_{2}-2 k-1}\left[\left(\xi_{1} \xi_{2}\right)-\left(\bar{\xi}_{1} \bar{\xi}_{2}\right)\right]^{2 k+1} .
\end{aligned}
$$

The right hand side of this equation can be brought to the form

$$
\begin{aligned}
\frac{\tilde{c}_{0}}{c_{0}} \sum_{k=0}^{s_{2}-1} \sum_{i=0}^{k} \sum_{j=0}^{k-i} \frac{(-1)^{j+k} 2^{s_{1}-s_{2}+1} i^{s_{1}+s_{2}} \Gamma\left(-2 k+2 s_{1}+2 s_{2}-1\right) \Gamma\left(-i+k+s_{1}-s_{2}+\frac{3}{2}\right)}{\sqrt{\pi} i ! j ! \Gamma\left(2 s_{1}\right) \Gamma\left(-i+2 s_{1}+1\right) \Gamma\left(2 s_{2}-2 k\right) \Gamma(-i-j+k+1)} \\
\times \frac{S_{3} Q_{1}^{s_{1}-s_{2}} P_{12}^{2 i+2 j-2 k+2 s_{2}-1}\left(P_{12}^{2}-Q_{1} Q_{2}\right)^{-i-j+k}}{\mathrm{x}_{23}^{2} \mathrm{x}_{13}^{2}}
\end{aligned}
$$

As before, this expression can be supplemented with the $s_{2}>s_{1}$ contribution, extended to diagonal $s_{1}=s_{2}$, then summed over spins as to build a generating function, see appendix B.2, giving the result:

$$
-\frac{\tilde{c}_{0}}{c_{0}} \frac{\exp \left(\frac{i}{2} Q_{1}+\frac{i}{2} Q_{2}\right)}{\mathrm{x}_{23}^{2} \mathrm{x}_{13}^{2}} S_{3} \sin P_{12},
$$

and matches exactly the CFT three-point function $(2.31):^{21}$

$$
\left\langle J_{s_{1}}\left(\mathrm{x}_{1}, \eta_{1}\right) J_{s_{2}}\left(\mathrm{x}_{2}, \eta_{2}\right) J_{0}\left(\mathrm{x}_{3}\right)\right\rangle_{h . s .}^{\Delta=2, \theta=\frac{\pi}{2}}=\left\langle J_{s_{1}}\left(\mathrm{x}_{1}, \eta_{1}\right) J_{s_{2}}\left(\mathrm{x}_{2}, \eta_{2}\right) J_{0}\left(\mathrm{x}_{3}\right)\right\rangle_{\text {f.f. }} .
$$

where we recall that the prefactor accounts for the difference in the boundary conditions for the scalar field and is equal to -2 .

\footnotetext{
${ }^{21}$ We again note that the answer is formally for the free fermion with leftover $\mathrm{U}(M)$ global symmetry.
} 


\subsubsection{Type-A, critical boson}

We take the $\Delta=1$ Type-A expression (6.29) and simply set $\Delta=2$. This gives

$$
\begin{aligned}
& \left\langle J_{s_{1}}\left(\mathrm{x}_{1}, \eta_{1}\right) J_{s_{2}}\left(\mathrm{x}_{2}, \eta_{2}\right) J_{0}\left(\mathrm{x}_{3}\right)\right\rangle_{h . s .}^{\Delta=2, \theta=0}=(-4 A) c_{s_{1}} c_{s_{2}} \tilde{c}_{0} v_{s_{1}, s_{2}, 0} \times \\
& \quad \times \int \frac{d^{3} \mathrm{x} d z}{z^{4}} K_{1} K_{2} K_{3}^{2}\left(\xi_{1} F_{2} \bar{\xi}_{1}\right)^{s_{1}-s_{2}} \sum_{k} \frac{2}{4^{s_{2}}} C_{2 k}^{2 s_{2}}\left[\left(\xi_{1} \xi_{2}\right)+\left(\bar{\xi}_{1} \bar{\xi}_{2}\right)\right]^{2 s_{2}-2 k}\left[\left(\xi_{1} \xi_{2}\right)-\left(\bar{\xi}_{1} \bar{\xi}_{2}\right)\right]^{2 k} .
\end{aligned}
$$

The right hand side of this equation is evaluated to yield

$$
\begin{gathered}
\frac{\tilde{c}_{0}}{c_{0}} \sum_{k=0}^{s_{2}} \sum_{i=0}^{k} \sum_{j=0}^{k-i} \frac{(-1)^{k-j} 2^{s_{1}-s_{2}+1} i^{s_{1}+s_{2}} \Gamma\left(-2 k+2 s_{1}+2 s_{2}\right) \Gamma\left(-i+k+s_{1}-s_{2}+1\right)}{\sqrt{\pi} i ! j ! \Gamma\left(2 s_{1}\right) \Gamma\left(-i+2 s_{1}+1\right) \Gamma\left(-2 k+2 s_{2}+1\right) \Gamma\left(-i-j+k+\frac{1}{2}\right)} \\
\times \frac{Q_{1}^{s_{1}-s_{2}} P_{12}^{2\left(i+j-k+s_{2}\right)}\left(P_{12}^{2}-Q_{1} Q_{2}\right)^{-i-j+k}}{\mathrm{x}_{23}^{2} \mathrm{x}_{13}^{2}}
\end{gathered}
$$

The three-point function in the CS-fermion theory was found in a form of recurrence relations in [25] (see also section 2.4). We find that the triple sum above is an explicit solution to those recursion relations. The leading coefficient is

$$
\left\langle J_{s_{1}}\left(\mathrm{x}_{1}, \eta_{1}\right) J_{s_{2}}\left(\mathrm{x}_{2}, \eta_{2}\right) J_{0}\left(\mathrm{x}_{3}\right)\right\rangle_{h . s .}^{\Delta=2, \theta=0}=-\frac{1}{2} \frac{\tilde{c}_{0}}{c_{0}} \frac{i^{s_{1}+s_{2}} 2^{-s_{1}-s_{2}+2}}{\Gamma\left(s_{1}+\frac{1}{2}\right) \Gamma\left(s_{2}+\frac{1}{2}\right)} \frac{Q_{1}^{s_{1}} Q_{2}^{s_{2}}}{\mathrm{x}_{23}^{2} \mathrm{x}_{13}^{2}}+\mathcal{O}\left(P_{12}\right) .
$$

The sum (6.38) can be evaluated explicitly, see appendix B.3. The computation shows that it is more convenient to use the $S_{3}$ variable. Indeed, the bulk integral gives $S_{3}$ after $O_{12}$ is applied. We expect this to be the case for the most general case of three non-zero spins too: it may be more convenient to express even structures in terms of even functions of the odd $S$ structures.

\subsubsection{Type-B, critical fermion}

Using the same technique as before we can compute the parity odd integral for $\Delta=1$, which for $\Delta=2$ reproduced the free fermion result, the only modification required being $K_{3}^{2} \rightarrow K_{3}$ :

$$
\begin{aligned}
& \left\langle J_{s_{1}}\left(\mathrm{x}_{1}, \eta_{1}\right) J_{s_{2}}\left(\mathrm{x}_{2}, \eta_{2}\right) J_{0}\left(\mathrm{x}_{3}\right)\right\rangle_{h . s .}^{\Delta=1, \theta=\frac{\pi}{2}}=(-4 A) c_{s_{1}} c_{s_{2}} c_{0} v_{s_{1}, s_{2}, 0} \times \\
& \times \int \frac{d^{3} \mathrm{x} d z}{z^{4}} K_{1} K_{2} K_{3}\left(\xi_{1} F_{2} \bar{\xi}_{1}\right)^{s_{1}-s_{2}} \sum_{k} \frac{2 i}{4^{s_{2}}} C_{2 k+1}^{2 s_{2}}\left[\left(\xi_{1} \xi_{2}\right)+\left(\bar{\xi}_{1} \bar{\xi}_{2}\right)\right]^{2 s_{2}-2 k-1}\left[\left(\xi_{1} \xi_{2}\right)-\left(\bar{\xi}_{1} \bar{\xi}_{2}\right)\right]^{2 k+1} .
\end{aligned}
$$

As a result, the right hand side of this equation yields the following triple sum representation for the critical fermion:

$$
\begin{aligned}
\sum_{k=0}^{s_{2}-1} \sum_{i=0}^{k} \sum_{j=0}^{k-i} \frac{(-1)^{j+k} i^{s_{1}+s_{2}} 2^{s_{1}-s_{2}+1} \Gamma\left(-2 k+2 s_{1}+2 s_{2}-1\right) \Gamma\left(-i+k+s_{1}-s_{2}+1\right)}{\sqrt{\pi} i ! j ! \Gamma\left(2 s_{1}\right)\left(-2 k+2 s_{2}-1\right) ! \Gamma\left(-i+2 s_{1}+1\right) \Gamma\left(-i-j+k+\frac{3}{2}\right)} \\
\times \frac{S_{3} Q_{1}^{s_{1}-s_{2}} P_{12}^{2 i+2 j-2 k+2 s_{2}-1}\left(P_{12}^{2}-Q_{1} Q_{2}\right)^{-i-j+k}}{\mathrm{x}_{12} \mathrm{x}_{13} \mathrm{x}_{23}}
\end{aligned}
$$


In [25], see also section 2.5, the generating function of the correlators was found in CSboson theory in an implicit form of a recurrence relation. The triple sum above provides an explicit solution to this system. The leading coefficient is easy to find, and gives

$$
\left\langle J_{s_{1}}\left(\mathrm{x}_{1}, \eta_{1}\right) J_{s_{2}}\left(\mathrm{x}_{2}, \eta_{2}\right) J_{0}\left(\mathrm{x}_{3}\right)\right\rangle_{h . s .}^{\Delta=1, \theta=\frac{\pi}{2}}=-\frac{i^{s_{1}+s_{2}} 2^{-s_{1}-s_{2}+2}}{\Gamma\left(s_{1}+\frac{1}{2}\right) \Gamma\left(s_{2}+\frac{1}{2}\right)} \frac{Q_{1}^{s_{1}-1} Q_{2}^{s_{2}-1} S_{3} P_{12}}{\mathrm{x}_{12} \mathrm{x}_{23} \mathrm{x}_{13}}+\mathcal{O}\left(P_{12}^{2}\right) .
$$

The sum (6.41) can be evaluated explicitly, see appendix B.4.

\subsubsection{Summary}

Combining all the four cases together with the $\theta$ dependence (6.28) we have confirmed that the structure of $\left\langle j_{s_{1}} j_{s_{2}} j_{0}\right\rangle$ is in accordance with the CFT result (2.36):

$$
\begin{aligned}
& \left\langle j_{s_{1}} j_{s_{2}} j_{0}\right\rangle_{B .}=\tilde{N}\left[\cos \theta\left\langle j_{s_{1}} j_{s_{2}} j_{0}\right\rangle_{f . b .}+\sin \theta\left\langle j_{s_{1}} j_{s_{2}} j_{0}\right\rangle_{\text {odd }}\right] \\
& \left\langle j_{s_{1}} j_{s_{2}} \tilde{j}_{0}\right\rangle_{F .}=\tilde{N}\left[\cos \theta\left\langle j_{s_{1}} j_{s_{2}} \tilde{j}_{0}\right\rangle_{f . f .}+\sin \theta\left\langle j_{s_{1}} j_{s_{2}} \tilde{j}_{0}\right\rangle_{\text {odd }}\right] .
\end{aligned}
$$

Here we restored the bulk coupling constant $G=1 / \tilde{N}$. The $\theta$-dependence results from the fact that the (anti)-selfdual HS Weyl tensors are identified with the order $s$-curls of the Fronsdal field with a phase shift (3.11)

$$
\begin{aligned}
& C_{\alpha_{1} \ldots \alpha_{2 s}}=e^{+i \theta} \nabla_{\alpha_{1}}^{\dot{\alpha}_{1}} \ldots \nabla_{\alpha_{s}}^{\dot{\alpha}_{s}} \phi_{\alpha_{s+1} \ldots \alpha_{2 s}, \dot{\alpha}_{1} \ldots \dot{\alpha}_{s}}, \\
& C_{\dot{\alpha}_{1} \ldots \dot{\alpha}_{2 s}}=e^{-i \theta} \nabla^{\alpha_{1}} \dot{\alpha}_{1} \ldots \nabla^{\alpha_{s}} \dot{\alpha}_{s} \phi_{\alpha_{1} \ldots \alpha_{s}, \dot{\alpha}_{s+1} \ldots \dot{\alpha}_{2 s}} .
\end{aligned}
$$

Given the $\theta$-dependence, the four structures in (6.43) can be found by evaluating the bulk integral for $\Delta=1,2$ and $\theta=0, \pi / 2$, as we did.

\section{Discussion}

We have extracted the full structure of the correlators that follow from the $\omega C$-correction to the scalar field equation: backreaction of $s_{1}$ and $s_{2}$ onto the $s=0$ field. The perfect agreement with the CFT results is found, including the $\theta$-dependence and the parityviolating structures, which were recently derived in [25] on the CFT side.

The most general bulk result includes the case of the gauged $\mathrm{U}(N)$ symmetry with the leftover $\mathrm{U}(M)$ global symmetry on the CFT side and should correspond to an extension of [11] to these cases. The results allow us to fix a part of the cubic action $s_{1}-s_{2}-0$ that is $\theta$-dependent. Indeed, upon treating various components of $\omega$ and $C$ as a bookkeeping device for the derivatives of the Fronsdal fields, the vertex has a rather simple form (schematically):

$$
V=v_{s_{1}, s_{2}, 0}^{\prime} \int \phi \omega^{\alpha\left(s_{1}+s_{2}-1\right), \dot{\alpha}\left(s_{1}-s_{2}-1\right)} C_{\alpha\left(2 s_{2}+s_{1}-s_{2}\right), \dot{\alpha}\left(s_{1}-s_{2}\right)} \hat{h}^{\alpha \dot{\alpha}}+h . c .,
$$

where $\hat{h}^{\alpha \dot{\alpha}}$ is the basis three-form. This expression, after plugging the propagators in place of $\omega$ and $C$ results in (6.1), i.e. the integral we have computed. The spin-dependent part of the coupling $v_{s_{1}, s_{2}, 0}^{\prime}$ is fixed by the HS algebra structure constants via $\omega \star C-C \star \tilde{\omega}$. Therefore, this coupling does not require anything beyond the HS algebra, in particular it 
does not rely on the Vasiliev equations, though they can, of course, be derived from them as well. The coupling is local and for that reason is easy to deal with.

The gauge invariance of the action or equations of motion implies that the correlation functions are those of conserved currents, see e.g. [54, 55]. This is the same statement as the invariance of the $S$-matrix of massless fields under the free gauge transformations. The bulk proof of this result heavily relies upon neglecting possible boundary terms. However, the CFT correlators are made of several structures: those given by free theories do correspond to conserved currents, while the parity-odd structures are not conserved when the triangle inequality is violated, e.g. $s_{1}+s_{2}<s_{3}$. The non-conservation of a current can be accounted for only by non-vanishing boundary terms [24] in the approach based on action principle. Therefore, the agreement for the parity-violating correlators cannot be explained by simple gauge invariance arguments and should be regarded as a test of the duality. Another result $[56,57]$ is that the duality with the critical boson/fermion ${ }^{22}$ should follow order by order in $1 / N$ expansion from the duality with the free boson/fermion. While for $\theta=0, \pi / 2$ this implies that the bulk results for critical models are not independent from those for the free models, it is unclear whether this argument can be extended to the full range of $\theta$ as on the CFT side the dependence on $\theta$ results from a highly nontrivial computation [11].

In this paper we could not extract the correlators for general three spins due to nonlocalities present in the additional $C C$-terms $[27,29,33]$ that need to be taken into account. These non-localities make the coefficient of the bulk vertex infinite, resulting in infinite correlators. The infinity is due to the presence of infinitely many of higher derivative copies of the same vertex that are stacked in the $C C$-terms. The divergences at tree level must not arise in any field theory, including HS theories. ${ }^{23}$ Indeed, the correlation functions on the CFT side are finite. Moreover, there is a one-to-one correspondence between all possible three-point correlators and cubic vertices in AdS, which allows one to manufacture an action up to the cubic terms that will yield any given three-point correlation functions and no infinities can arise. This was explicitly done for some of the cubic vertices in $[28,58,59]$ (full cubic action of Type-A in any $d$ was obtained in [59]), see also [60-62] for the quartic results. In the recent paper [34] it was conjectured how to modify the $C C$-terms as to make them local. The field redefinition that does the job is non-local and changes the coefficient of the correlator from an infinite number to a finite one. Moreover, the $C C$ terms correspond to the abelian vertices at this order (in the sense that are not fixed by the HS symmetry) and can in principle be arbitrary. Also, non-local redefinitions can result in any given coefficient [33, 63-66]. Nonetheless, the proposal of [34] involves a specific type of field redefinition, and as such it can be treated as a conjecture. Taking this point of view, we have tested this redefinition, which involves the $C C$ terms, and we have found that it does produce an answer for the spin $0-s-s$ amplitude that agrees with the CFT result. Further tests of this conjecture will require the study of more three-point functions, namely those involving three arbitrary spins, and then of higher order amplitudes, which may possibly require higher order extension of the proposed field redefinition as well.

\footnotetext{
${ }^{22}$ In [56] the duality between Type-A HS theory and free/critical boson was studied, the extension to Type-B vs. free/critical fermion should be straightforward.

${ }^{23}$ There exist extremal correlators and the divergences observed in $[27,29,33]$ have nothing to do with those.
} 
It is interesting that the $\theta$ dependence enters the Vasiliev equations in a very simple way $-e^{ \pm i \theta}$ factors in two terms in the master equations. It was proved in [67] that the $\theta$-deformation is indeed unique in HS gravity. In the perturbation theory this leads to some proliferation of $\theta$-dependent terms, so that in the $\omega$-equation of motion one generally finds terms from $e^{-i n \theta}$ to $e^{+i n \theta}$ at the $n$-th order. Some of them are too non-local to be treated by field theory methods. Hopefully, there are simple enough non-local redefinitions that render the couplings finite and do not introduce any spin-dependent $\theta$-coefficients. This would imply that the planar $n$-point correlation functions of HS currents in Chern-Simons matter theories have a form similar to those of the bulk three-point amplitudes even for $n>3$ : a number of structures multiplied by simple factors ranging from $\cos ^{n} \theta$ to $\sin ^{n} \theta$ at the level of $n$-point functions. It would be very interesting to check whether such a simple form arises for the $n$-point functions on the both sides of the duality.

Note added: after the completion of our work, we learned that V.E. Didenko and M. A. Vasiliev are in the process of publishing results [69] on problems closely related to the subject matter of this paper.

\section{Acknowledgments}

We would like to thank Simone Giombi, Shiroman Prakash, Per Sundell and Sasha Zhiboedov for the very useful discussions and comments. E.Sk is grateful to Simone Giombi, V. Gurucharan, Volodya Kirilin and Shiroman Prakash for the very useful discussions in the course of collaboration [25]. The work of E.Se. and Y.Z. is supported in part by NSF grant PHY-1214344, in part by the Mitchell Institute for Fundamental Physics and Astronomy and in part by Conicyt MEC grant PAI 80160107. The work of E.Sk. was supported in part by the Russian Science Foundation grant 14-42-00047 in association with Lebedev Physical Institute and by the DFG Transregional Collaborative Research Centre TRR 33 and the DFG cluster of excellence "Origin and Structure of the Universe". E.Se. thanks Pontifica Universidad Católica de Valparaiso for hospitality. E.Sk. thanks the Galileo Galilei Institute for Theoretical Physics (GGI) for the hospitality and INFN for partial support during the completion of this work, within the program "New Developments in $A d S_{3} / C F T_{2}$ Holography".

\section{A Notation and conventions}

We adopt the mostly plus convention for the metric $\eta_{\underline{m n}}=(-+++)$, which makes the Euclidean rotation easier to implement. Choosing $x^{\underline{m}}=(\overrightarrow{\mathrm{x}}, z)$ to be Poincaré coordinates with $z$ being the radial coordinate and $\overrightarrow{\mathrm{x}}$ the three coordinates along the boundary, the $A d S_{4}$-background can be described by vierbein $h_{\underline{m}}^{\alpha \dot{\alpha}}$ and spin-connection that splits into (anti) self-dual parts $\omega_{(0) \underline{m}^{\alpha \beta}}$ and $\bar{\omega}_{(0) \underline{m}}{ }^{\dot{\alpha} \dot{\beta}}$ :

$$
h^{\alpha \dot{\alpha}}=\frac{1}{2 z} \sigma_{\underline{m}}^{\alpha \dot{\alpha}} d x^{\underline{m}}, \quad \omega_{(0)}^{\alpha \beta}=\frac{i}{2 z} \vec{\sigma}^{\alpha \beta} \cdot d \vec{x}, \quad \bar{\omega}_{(0)}^{\dot{\alpha} \dot{\beta}}=-\frac{i}{2 z} \vec{\sigma}^{\alpha \beta} \cdot d \vec{x} .
$$


The matrices $\sigma_{\underline{m}}^{\alpha \dot{\alpha}}$ are constant and in our convention they are given by $\sigma_{\underline{m}}^{\alpha \dot{\beta}}=\left(\vec{\sigma}^{\alpha \beta}, i \epsilon^{\alpha \beta}\right)$. We have the relations ${ }^{24}$

$$
\begin{array}{rlrl}
\mathrm{x}^{\alpha \beta} & =\vec{\sigma}^{\alpha \beta} \cdot \overrightarrow{\mathrm{x}}, & \mathrm{x}^{2}=-\frac{1}{2} \mathrm{x}^{\alpha \beta} \mathrm{x}_{\alpha \beta}, & \sigma_{\underline{m}}^{\alpha \dot{\alpha}} \sigma_{\underline{n} \alpha \dot{\alpha}}=-2 \eta_{\underline{m n}}, \\
x^{\alpha \dot{\alpha}}=\mathrm{x}^{\alpha \dot{\alpha}}+i z \epsilon^{\alpha \dot{\alpha}}, & x^{2}=\mathrm{x}^{2}+z^{2}, & \mathrm{x}_{i j}=\left|\mathrm{x}_{i}-\mathrm{x}_{j}\right| .
\end{array}
$$

The inverse vierbein $h_{\alpha \dot{\alpha}}^{\frac{m}{\alpha}}=-z \sigma \frac{m}{\alpha \dot{\alpha}}$ obeys the relations

$$
h_{\underline{m}}^{\alpha \dot{\alpha}} h_{\alpha \dot{\alpha}}^{\underline{n}}=\delta_{\underline{m}}^{\frac{n}{m}}, \quad h_{\underline{m}}^{\alpha \dot{\alpha}} h_{\beta \dot{\beta}}^{\underline{m}}=\delta_{\beta}^{\alpha} \delta_{\dot{\beta}}^{\dot{\alpha}},
$$

and the $A d S_{4}$ metric tensor and spin connection are given by

$$
\begin{aligned}
g_{\underline{m n}} & =-h_{\underline{m}}^{\alpha \dot{\alpha}} h_{\underline{n} \alpha \dot{\alpha}} d x^{\underline{m}} d x^{\underline{n}}=\frac{1}{2 z^{2}} \eta_{\underline{m n}} d x^{\underline{m}} d x^{\underline{n}}, \\
\Omega & =\frac{1}{4 i}\left[\omega_{(0) \alpha \beta} y^{\alpha} y^{\beta}+\bar{\omega}_{(0) \dot{\alpha} \dot{\beta}} \bar{y}^{\dot{\alpha}} y^{\dot{\beta}}+2 h_{\alpha \dot{\beta}} y^{\alpha} \bar{y}^{\dot{\beta}}\right] .
\end{aligned}
$$

We use the raising and lowering conventions: $X^{\alpha}=\epsilon^{\alpha \beta} X_{\beta}$ and $X_{\alpha}=X^{\beta} \epsilon_{\beta \alpha}$ with $\epsilon_{\alpha \gamma} \epsilon^{\beta \gamma}=$ $\delta_{\alpha}^{\beta}$, and similar conventions for the dotted indices. We use the convention $\epsilon_{12}=-\epsilon_{21}=1$. It is sometimes convenient to define $\nabla_{\alpha \dot{\alpha}}$ as $\nabla=\nabla_{\alpha \dot{\alpha}} h_{\underline{m}}^{\alpha \dot{\alpha}} d x \underline{\underline{m}}$.

\section{B Triple sums}

Here we present some details on the triple sums that result from the bulk integrals.

\section{B.1 Type-A, free boson}

We begin with the evaluation of the triple sum (6.30). Expanding the last factor in (6.30), we obtain

$$
\begin{aligned}
\sum_{l=0}^{s_{2}} \sum_{k=l}^{s_{2}} \sum_{i=0}^{k-l} \sum_{j=0}^{k-l-i} \frac{(-1)^{k+j+l} i^{s_{1}+s_{2}} 2^{s_{1}-s_{2}+1} \Gamma\left(2\left(-k+s_{1}+s_{2}\right)\right) \Gamma\left(-i+k+s_{1}-s_{2}+\frac{1}{2}\right)}{\sqrt{\pi} i ! j ! l !\left(2 s_{1}-1\right) !\left(2 s_{2}-2 k\right) !\left(-i+2 s_{1}\right) !(k-i-j-l) !} \\
\quad \times \frac{Q_{1}^{s_{1}} Q_{2}^{s_{2}}\left(\frac{P_{12}^{2}}{Q_{1} Q_{2}}\right)^{s_{2}-l}}{\mathrm{x}_{12} \mathrm{x}_{13} \mathrm{x}_{23}} .
\end{aligned}
$$

After the first summation over $j$, the coefficient is proportional to ${ }_{1} F_{0}(i-k+l, 1)$. Since $k \geq i+l$, and ${ }_{1} F_{0}(n, 1)$ vanishes when $n$ is a negative integer, the second sum over $i$ is equivalent to simply substituting $i \rightarrow k-l$. At this point we have

$$
\sum_{l=0}^{s_{2}} \sum_{k=l}^{s_{2}} \frac{(-1)^{k+l} i^{s_{1}+s_{2}} 2^{s_{1}-s_{2}+1} \Gamma\left(2\left(-k+s_{1}+s_{2}\right)\right) \Gamma\left(l+s_{1}-s_{2}+\frac{1}{2}\right)}{\sqrt{\pi} l !\left(2 s_{1}-1\right) !\left(2 s_{2}-2 k\right) !\left(-k+l+2 s_{1}\right) !(k-l) !} \times \frac{Q_{1}^{s_{1}} Q_{2}^{s_{2}}\left(\frac{P_{12}^{2}}{Q_{1} Q_{2}}\right)^{s_{2}-l}}{\mathrm{x}_{12} \mathrm{x}_{13} \mathrm{x}_{23}} .
$$

\footnotetext{
${ }^{24}$ The bulk objects may transform under the Lorentz algebra, $s l(2, \mathbb{C})$, while the choice of the Poincaré coordinates breaks the manifest symmetry down to the boundary Lorentz algebra, $\operatorname{sl}(2, \mathbb{R})$. In particular, there exists $\epsilon^{\alpha \dot{\alpha}}$ that allows one to map between dotted and undotted indices. For that reason the $3 d$ coordinates $\mathrm{x}$ can carry different types of indices $\mathrm{x}^{\alpha \beta}, \mathrm{x}^{\alpha \dot{\beta}}$ or $\mathrm{x}^{\dot{\alpha} \dot{\beta}}$, while being the same object.
} 
We then evaluate the summation over $k$ and obtain

$$
\begin{aligned}
& \sum_{l=0}^{s_{2}} \frac{i^{s_{1}+s_{2}} 2^{s_{1}-s_{2}+1} \Gamma\left(2\left(-l+s_{1}+s_{2}\right)\right) \Gamma\left(l+s_{1}-s_{2}+\frac{1}{2}\right)}{\sqrt{\pi} l !\left(2 s_{1}-1\right) !\left(2 s_{1}\right) !\left(2 s_{2}-2 l\right) !} \\
& \times{ }_{3} F_{2}\left(-2 s_{1}, l-s_{2}, \frac{1}{2}+l-s_{2} ; \frac{1}{2}+l-s_{1}-s_{2}, 1+l-s_{1}-s_{2} ; 1\right) \times \frac{Q_{1}^{s_{1}} Q_{2}^{s_{2}}\left(\frac{P_{12}^{2}}{Q_{1} Q_{2}}\right)^{s_{2}-l}}{\mathrm{x}_{12} \mathrm{x}_{13} \mathrm{x}_{23}} .
\end{aligned}
$$

Using the identity

$$
{ }_{3} F_{2}(-n, b, c ; d, b+c-d-n+1 ; 1)=\frac{(d-b)_{n}(d-c)_{n}}{(d)_{n}(d-b-c)_{n}}, \quad n \in \mathbb{N}
$$

where $(a)_{n}=a(a+1) \cdots(a+n-1)$ is the Pochhammer symbol, the hypergeometric function can then be written in terms of gamma functions

$$
\begin{aligned}
& { }_{3} F_{2}\left(-2 s_{1}, l-s_{2}, \frac{1}{2}+l-s_{2} ; \frac{1}{2}+l-s_{1}-s_{2}, 1+l-s_{1}-s_{2} ; 1\right) \\
& =\frac{\left(\frac{1}{2}-s_{1}\right)_{2 s_{1}}\left(-s_{1}\right)_{2 s_{1}}}{\left(\frac{1}{2}+l-s_{1}-s_{2}\right)_{2 s_{1}}\left(-l-s_{1}+s_{2}\right)_{2 s_{1}}}=\frac{\Gamma\left(2 s_{1}\right) \Gamma\left(1+2 s_{1}\right)}{\Gamma\left(1+2 l+2 s_{1}-2 s_{2}\right) \Gamma\left(2\left(-l+s_{1}+s_{2}\right)\right)} .
\end{aligned}
$$

Note that $s_{1} \geq s_{2} \geq l$, and a cancellation of zeros in $(-s)_{2 s_{1}}$ and $\left(-l-s_{1}+s_{2}\right)_{2 s_{1}}$ has been performed to get the bottom line. Replacing the hypergeometric function by the above expression, one can see that the coefficients in (B.3) are equal to the ones from the CFT side, cf. (2.20).

\section{B.2 Type-B, free fermion}

We now consider the triple sum (6.34), which upon the expansion of the last factor, can be written as

$$
\begin{gathered}
\sum_{l=0}^{s_{2}-1} \sum_{k=l}^{s_{2}-1} \sum_{i=0}^{k-l} \sum_{j=0}^{k-l-i} \frac{(-1)^{k+j+l} i^{s_{1}+s_{2}} 2^{s_{1}-s_{2}+1} \Gamma\left(2\left(-k+s_{1}+s_{2}\right)-1\right) \Gamma\left(-i+k+s_{1}-s_{2}+\frac{3}{2}\right)}{\sqrt{\pi} i ! j ! l !\left(2 s_{1}-1\right) !\left(2 s_{2}-2 k-1\right) !\left(-i+2 s_{1}\right) !(k-i-j-l) !} \\
\times \frac{S_{3} Q_{1}^{s_{1}} Q_{2}^{s_{2}}\left(\frac{P_{12}^{2}}{Q_{1} Q_{2}}\right)^{s_{2}-l}\left(P_{12}\right)^{-1}}{\mathrm{x}_{23}^{2} \mathrm{x}_{13}^{2}} .
\end{gathered}
$$

As in the type-A model case, after the first summation over $j$, the coefficient of each term is proportional to ${ }_{1} F_{0}(i-k+l, 1)$, therefore for the same reason the second summation over $i$ is equivalent to the substitution $i \rightarrow k-l$ in the summand. The result is then

$$
\begin{aligned}
\sum_{l=0}^{s_{2}-1} \sum_{k=l}^{s_{2}-1} & \frac{(-1)^{k+l} i^{s_{1}+s_{2}} 2^{s_{1}-s_{2}+1} \Gamma\left(2\left(-k+s_{1}+s_{2}\right)-1\right) \Gamma\left(l+s_{1}-s_{2}+\frac{3}{2}\right)}{\sqrt{\pi} l !\left(2 s_{1}-1\right) !\left(2 s_{2}-2 k-1\right) !\left(-k+l+2 s_{1}\right) !(k-l) !} \\
& \times \frac{S_{3} Q_{1}^{s_{1}} Q_{2}^{s_{2}}\left(\frac{P_{12}^{2}}{Q_{1} Q_{2}}\right)^{s_{2}-l}\left(P_{12}\right)^{-1}}{\mathrm{x}_{23}^{2} \mathrm{x}_{13}^{2}} .
\end{aligned}
$$


We sum over $k$ and obtain

$$
\begin{aligned}
& \sum_{l=0}^{s_{2}-1} \frac{i^{s_{1}+s_{2}} 2^{s_{1}-s_{2}+1} \Gamma\left(2\left(-l+s_{1}+s_{2}\right)-1\right) \Gamma\left(l+s_{1}-s_{2}+\frac{3}{2}\right)}{\sqrt{\pi} l !\left(2 s_{1}-1\right) !\left(2 s_{1}\right) !\left(2 s_{2}-2 l-1\right) !} \\
& \quad \times{ }_{3} F_{2}\left(-2 s_{1}, \frac{1}{2}+l-s_{2}, 1+l-s_{2} ; 1+l-s_{1}-s_{2}, \frac{3}{2}+l-s_{1}-s_{2} ; 1\right) \\
& \quad \times \frac{S_{3} Q_{1}^{s_{1}} Q_{2}^{s_{2}}\left(\frac{P_{12}^{2}}{Q_{1} Q_{2}}\right)^{s_{2}-l}\left(P_{12}\right)^{-1}}{\mathrm{x}_{23}^{2} \mathrm{x}_{13}^{2}} .
\end{aligned}
$$

Using the fact that the hypergeometric function in the above expression equals

$$
\frac{\Gamma\left(2 s_{1}\right) \Gamma\left(2 s_{1}+1\right)}{\Gamma\left(2 s_{1}-2 s_{2}+2 l+2\right) \Gamma\left(2 s_{1}+2 s_{2}-2 l-1\right)},
$$

we find that the coefficient of each term in (B.8) matches the corresponding one from CFT three-point function exactly, cf. (2.31).

\section{B.3 Type-A, critical boson}

Next, we evaluate the summations in (6.38). This can be done in an easy way by keeping the invariant structure $S_{3}$ instead of $Q_{2}$ :

$$
\begin{gathered}
\frac{\tilde{c}_{0}}{c_{0}} \sum_{k=0}^{s_{2}} \sum_{i=0}^{k} \sum_{j=0}^{k-i} \frac{(-1)^{k-j} 2^{s_{1}-s_{2}+1} i^{s_{1}+s_{2}} \Gamma\left(-2 k+2 s_{1}+2 s_{2}\right) \Gamma\left(-i+k+s_{1}-s_{2}+1\right)}{\sqrt{\pi} i ! j ! \Gamma\left(2 s_{1}\right) \Gamma\left(-i+2 s_{1}+1\right) \Gamma\left(-2 k+2 s_{2}+1\right) \Gamma\left(-i-j+k+\frac{1}{2}\right)} \\
\times \frac{\left(Q_{1}\right)^{s_{1}-s_{2}}\left(P_{12}\right)^{2\left(i+j-k+s_{2}\right)}\left(\left(S_{3}\right)^{2}\right)^{-i-j+k}}{\mathrm{x}_{23}^{2} \mathrm{x}_{13}^{2}}
\end{gathered}
$$

For the $\left(S_{3}\right)^{2}$ term with power $l=k-i-j$, we have

$$
\begin{gathered}
\frac{\tilde{c}_{0}}{c_{0}} \sum_{l=0}^{s_{2}} \sum_{k=l}^{s_{2}} \sum_{j=0}^{k-l} \frac{(-1)^{k-j} 2^{s_{1}-s_{2}+1} i^{s_{1}+s_{2}} \Gamma\left(-2 k+2 s_{1}+2 s_{2}\right) \Gamma\left(l+j+s_{1}-s_{2}+1\right)}{\sqrt{\pi}(k-l-j) ! j ! \Gamma\left(2 s_{1}\right) \Gamma\left(-k+l+j+2 s_{1}+1\right) \Gamma\left(-2 k+2 s_{2}+1\right) \Gamma\left(l+\frac{1}{2}\right)} \\
\times \frac{\left(Q_{1}\right)^{s_{1}-s_{2}}\left(P_{12}\right)^{2\left(s_{2}-l\right)}\left(S_{3}\right)^{2 l}}{\mathrm{x}_{23}^{2} \mathrm{x}_{13}^{2}}
\end{gathered}
$$

Notice that the summation over $i$ is now replaced by $i \rightarrow k-l-j$. After the first summation we obtain

$$
\begin{aligned}
\frac{\tilde{c}_{0}}{c_{0}} \sum_{k=l}^{s_{2}} & \frac{(-1)^{k} i^{s_{1}+s_{2}} 2^{1+s_{1}-s_{2}} \Gamma\left(1+l+s_{1}-s_{2}\right) \Gamma\left(2\left(-k+s_{1}+s_{2}\right)\right)}{\sqrt{\pi} \Gamma(1+k-l) \Gamma\left(\frac{1}{2}+l\right) \Gamma\left(2 s_{1}\right) \Gamma\left(1-2 k+2 s_{2}\right) \Gamma\left(1-k+l+2 s_{1}\right)} \\
& \times{ }_{2} F_{1}\left(-k+l, 1+l+s_{1}-s_{2}, 1-k+l+2 s_{1}, 1\right) \times \frac{\left(Q_{1}\right)^{s_{1}-s_{2}}\left(P_{12}\right)^{2\left(s_{2}-l\right)}\left(S_{3}\right)^{2 l}}{\mathrm{x}_{23}^{2} \mathrm{x}_{13}^{2}} .
\end{aligned}
$$

Replacing ${ }_{2} F_{1}\left(-k+l, 1+l+s_{1}-s_{2}, 1-k+l+2 s_{1}, 1\right)$ by

$$
\frac{\Gamma\left(1-k+l+2 s_{1}\right) \Gamma\left(s_{1}+s_{2}-l\right)}{\Gamma\left(1+2 s_{1}\right) \Gamma\left(s_{1}+s_{2}-k\right)},
$$


we can evaluate the second summation and the result is

$$
\begin{gathered}
\frac{\tilde{c}_{0}}{c_{0}} \sum_{l=0}^{s_{2}} \frac{(-1)^{l} i^{s_{1}+s_{2}} 2^{1+s_{1}-s_{2}} \Gamma\left(1+l+s_{1}-s_{2}\right) \Gamma\left(2\left(-l+s_{1}+s_{2}\right)\right)}{\sqrt{\pi} \Gamma\left(\frac{1}{2}+l\right) \Gamma\left(2 s_{1}\right) \Gamma\left(1+2 s_{1}\right) \Gamma\left(1-2 l+2 s_{2}\right)} \\
\quad \times{ }_{2} F_{1}\left(l-s_{2}, \frac{1}{2}+l-s_{2}, \frac{1}{2}+l-s_{1}-s_{2}, 1\right) \\
\quad \times \frac{\left(Q_{1}\right)^{s_{1}-s_{2}}\left(P_{12}\right)^{2\left(s_{2}-l\right)}\left(S_{3}\right)^{2 l}}{\mathrm{x}_{23}^{2} \mathrm{x}_{13}^{2}} \quad(\text { Type-A, } \Delta=2) .
\end{gathered}
$$

This formula explicitly gives the Taylor coefficients of the generating function for critical boson theory. To facilitate the comparison between Type-A models with different boundary conditions, we rewrite (6.30) in terms of $Q_{1}, P_{12}$ and $S_{3}$, and redo the summations, following the above procedure. The answer is

$$
\begin{aligned}
\sum_{l=0}^{s_{2}} & \frac{(-1)^{l} i^{s_{1}+s_{2}} 2^{1+s_{1}-s_{2}} \Gamma\left(\frac{1}{2}+l+s_{1}-s_{2}\right) \Gamma\left(2\left(-l+s_{1}+s_{2}\right)\right)}{\sqrt{\pi} \Gamma(1+l) \Gamma\left(2 s_{1}\right) \Gamma\left(1+2 s_{1}\right) \Gamma\left(1-2 l+2 s_{2}\right)} \\
& \times{ }_{2} F_{1}\left(l-s_{2}, \frac{1}{2}+l-s_{2}, 1+l-s_{1}-s_{2}, 1\right) \\
& \times \frac{\left(Q_{1}\right)^{s_{1}-s_{2}}\left(P_{12}\right)^{2\left(s_{2}-l\right)}\left(S_{3}\right)^{2 l}}{\mathrm{x}_{12} \mathrm{x}_{13} \mathrm{x}_{23}} \quad(\text { Type-A, } \Delta=1) .
\end{aligned}
$$

This is just (B.3) but written in terms of $Q_{1}, P_{12}$ and $S_{3}$ instead.

\section{B.4 Type-B, critical fermion}

Starting with (6.41), we use invariant structure $S_{3}$ and make the change of the index $i \rightarrow k-l-j$ :

$$
\begin{aligned}
\sum_{l=0}^{s_{2}-1} \sum_{k=l}^{s_{2}-1} \sum_{j=0}^{k-l} & \frac{(-1)^{j+k} i^{s_{1}+s_{2}} 2^{s_{1}-s_{2}+1} \Gamma\left(-2 k+2 s_{1}+2 s_{2}-1\right) \Gamma\left(l+j+s_{1}-s_{2}+1\right)}{\sqrt{\pi}(k-l-j) ! j ! \Gamma\left(2 s_{1}\right)\left(-2 k+2 s_{2}-1\right) ! \Gamma\left(l+j-k+2 s_{1}+1\right) \Gamma\left(l+\frac{3}{2}\right)} \\
& \times \frac{\left(Q_{1}\right)^{s_{1}-s_{2}}\left(P_{12}\right)^{2\left(s_{2}-l\right)-1}\left(S_{3}\right)^{2 l+1}}{\mathrm{x}_{12} \mathrm{x}_{13} \mathrm{x}_{23}}
\end{aligned}
$$

As in the case of Type-A with $\Delta=2$, it is straightforward to evaluate the first two sums. The result is

$$
\begin{gathered}
\sum_{l=0}^{s_{2}-1} \frac{(-1)^{l} i^{s_{1}+s_{2}} 2^{s_{1}-s_{2}+1} \Gamma\left(1+l+s_{1}-s_{2}\right) \Gamma\left(2 s_{1}+2 s_{2}-2 l-1\right)}{\sqrt{\pi} \Gamma\left(\frac{3}{2}+l\right) \Gamma\left(2 s_{1}\right) \Gamma\left(2 s_{1}+1\right) \Gamma\left(2 s_{2}-2 l\right)} \\
\quad \times{ }_{2} F_{1}\left(\frac{1}{2}+l-s_{2}, 1+l-s_{2}, \frac{3}{2}+l-s_{1}-s_{2}, 1\right) \\
\quad \times \frac{\left(Q_{1}\right)^{s_{1}-s_{2}}\left(P_{12}\right)^{2\left(s_{2}-l\right)-1}\left(S_{3}\right)^{2 l+1}}{\mathrm{x}_{12} \mathrm{x}_{13} \mathrm{x}_{23}} \quad(\text { Type-B }, \Delta=1) .
\end{gathered}
$$


One can also rewrite (B.8) in terms of $Q_{1}, P_{12}$ and $S_{3}$, and the result is

$$
\begin{gathered}
\sum_{l=0}^{s_{2}-1} \frac{(-1)^{l} i^{s_{1}+s_{2}} 2^{s_{1}-s_{2}+1} \Gamma\left(\frac{3}{2}+l+s_{1}-s_{2}\right) \Gamma\left(2 s_{1}+2 s_{2}-2 l-1\right)}{\sqrt{\pi} \Gamma(1+l) \Gamma\left(2 s_{1}\right) \Gamma\left(2 s_{1}+1\right) \Gamma\left(2 s_{2}-2 l\right)} \\
\quad \times{ }_{2} F_{1}\left(\frac{1}{2}+l-s_{2}, 1+l-s_{2}, 1+l-s_{1}-s_{2}, 1\right) \\
\times \frac{\left(Q_{1}\right)^{s_{1}-s_{2}}\left(P_{12}\right)^{2\left(s_{2}-l\right)-1}\left(S_{3}\right)^{2 l+1}}{\mathrm{x}_{23}^{2} \mathrm{x}_{13}^{2}} \quad(\text { Type-B, } \Delta=2) .
\end{gathered}
$$

Open Access. This article is distributed under the terms of the Creative Commons Attribution License (CC-BY 4.0), which permits any use, distribution and reproduction in any medium, provided the original author(s) and source are credited.

\section{References}

[1] I.R. Klebanov and A.M. Polyakov, AdS dual of the critical $O(N)$ vector model, Phys. Lett. B 550 (2002) 213 [hep-th/0210114] [INSPIRE].

[2] E. Sezgin and P. Sundell, Massless higher spins and holography, Nucl. Phys. B 644 (2002) 303 [Erratum ibid. B 660 (2003) 403] [hep-th/0205131] [INSPIRE].

[3] E. Sezgin and P. Sundell, Holography in 4D (super) higher spin theories and a test via cubic scalar couplings, JHEP 07 (2005) 044 [hep-th/0305040] [INSPIRE].

[4] R.G. Leigh and A.C. Petkou, Holography of the $N=1$ higher spin theory on $A d S_{4}$, JHEP 06 (2003) 011 [hep-th/0304217] [INSPIRE].

[5] J. Maldacena and A. Zhiboedov, Constraining conformal field theories with a higher spin symmetry, J. Phys. A 46 (2013) 214011 [arXiv:1112.1016] [INSPIRE].

[6] N. Boulanger, D. Ponomarev, E.D. Skvortsov and M. Taronna, On the uniqueness of higher-spin symmetries in AdS and CFT, Int. J. Mod. Phys. A 28 (2013) 1350162 [arXiv: 1305.5180] [INSPIRE].

[7] V. Alba and K. Diab, Constraining conformal field theories with a higher spin symmetry in

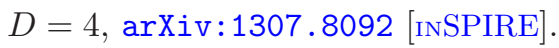

[8] V. Alba and K. Diab, Constraining conformal field theories with a higher spin symmetry in $d>3$ dimensions, JHEP 03 (2016) 044 [arXiv: 1510.02535] [INSPIRE].

[9] S. Giombi, S. Minwalla, S. Prakash, S.P. Trivedi, S.R. Wadia and X. Yin, Chern-Simons theory with vector fermion matter, Eur. Phys. J. C 72 (2012) 2112 [arXiv:1110.4386] [INSPIRE].

[10] O. Aharony, G. Gur-Ari and R. Yacoby, $D=3$ bosonic vector models coupled to Chern-Simons gauge theories, JHEP 03 (2012) 037 [arXiv:1110.4382] [INSPIRE].

[11] J. Maldacena and A. Zhiboedov, Constraining conformal field theories with a slightly broken higher spin symmetry, Class. Quant. Grav. 30 (2013) 104003 [arXiv: 1204.3882] [INSPIRE].

[12] S. Giombi and X. Yin, The higher spin/vector model duality, J. Phys. A 46 (2013) 214003 [arXiv: 1208.4036] [INSPIRE]. 
[13] S. Giombi, Higher spin-CFT duality, in Proceedings, Theoretical Advanced Study Institute in Elementary Particle Physics: New Frontiers in Fields and Strings, (TASI 2015), Boulder CO U.S.A. 1-26 June 2015, pg. 137 [arXiv:1607.02967] [INSPIRE].

[14] O. Aharony, G. Gur-Ari and R. Yacoby, Correlation functions of large- $N$ Chern-Simons-matter theories and bosonization in three dimensions, JHEP 12 (2012) 028 [arXiv:1207.4593] [INSPIRE].

[15] G. Gur-Ari and R. Yacoby, Correlators of large-N fermionic Chern-Simons vector models, JHEP 02 (2013) 150 [arXiv:1211.1866] [INSPIRE].

[16] O. Aharony, Baryons, monopoles and dualities in Chern-Simons-matter theories, JHEP 02 (2016) 093 [arXiv: 1512.00161] [INSPIRE].

[17] N. Seiberg, T. Senthil, C. Wang and E. Witten, A duality web in $2+1$ dimensions and condensed matter physics, Annals Phys. 374 (2016) 395 [arXiv:1606.01989] [INSPIRE].

[18] A. Karch and D. Tong, Particle-vortex duality from 3d bosonization, Phys. Rev. X 6 (2016) 031043 [arXiv:1606.01893] [INSPIRE].

[19] O. Aharony, S. Giombi, G. Gur-Ari, J. Maldacena and R. Yacoby, The thermal free energy in large-N Chern-Simons-matter theories, JHEP 03 (2013) 121 [arXiv:1211.4843] [INSPIRE].

[20] S. Jain, S. Minwalla, T. Sharma, T. Takimi, S.R. Wadia and S. Yokoyama, Phases of large- $N$ vector Chern-Simons theories on $S^{2} \times S^{1}$, JHEP 09 (2013) 009 [arXiv:1301.6169] [INSPIRE].

[21] S. Minwalla and S. Yokoyama, Chern Simons bosonization along RG flows, JHEP 02 (2016) 103 [arXiv: 1507.04546] [INSPIRE].

[22] M.A. Vasiliev, Properties of equations of motion of interacting gauge fields of all spins in $(3+1)$-dimensions, Class. Quant. Grav. 8 (1991) 1387 [INSPIRE].

[23] M.A. Vasiliev, Higher spin gauge theories: star product and AdS space, hep-th/9910096 [INSPIRE].

[24] S. Giombi, S. Prakash and X. Yin, A note on CFT correlators in three dimensions, JHEP 07 (2013) 105 [arXiv:1104.4317] [INSPIRE].

[25] S. Giombi, V. Gurucharan, V. Kirilin, S. Prakash and E. Skvortsov, On the higher-spin spectrum in large-N Chern-Simons vector models, JHEP 01 (2017) 058 [arXiv: 1610.08472] [INSPIRE].

[26] A.C. Petkou, Evaluating the AdS dual of the critical $O(N)$ vector model, JHEP 03 (2003) 049 [hep-th/0302063] [INSPIRE].

[27] S. Giombi and X. Yin, Higher spin gauge theory and holography: the three-point functions, JHEP 09 (2010) 115 [arXiv:0912.3462] [INSPIRE].

[28] X. Bekaert, J. Erdmenger, D. Ponomarev and C. Sleight, Towards holographic higher-spin interactions: four-point functions and higher-spin exchange, JHEP 03 (2015) 170 [arXiv:1412.0016] [INSPIRE].

[29] S. Giombi and X. Yin, Higher spins in AdS and twistorial holography, JHEP 04 (2011) 086 [arXiv: 1004.3736] [INSPIRE].

[30] E. Sezgin and P. Sundell, Geometry and observables in Vasiliev's higher spin gravity, JHEP 07 (2012) 121 [arXiv:1103.2360] [INSPIRE]. 
[31] N. Colombo and P. Sundell, Higher spin gravity amplitudes from zero-form charges, arXiv: 1208.3880 [INSPIRE].

[32] V.E. Didenko and E.D. Skvortsov, Exact higher-spin symmetry in CFT: all correlators in unbroken Vasiliev theory, JHEP 04 (2013) 158 [arXiv:1210.7963] [INSPIRE].

[33] N. Boulanger, P. Kessel, E.D. Skvortsov and M. Taronna, Higher spin interactions in four-dimensions: Vasiliev versus Fronsdal, J. Phys. A 49 (2016) 095402 [arXiv: 1508.04139] [INSPIRE].

[34] M.A. Vasiliev, Current interactions and holography from the 0-form sector of nonlinear higher-spin equations, arXiv: 1605.02662 [INSPIRE].

[35] M.A. Vasiliev, Consistent equation for interacting gauge fields of all spins in (3+1)-dimensions, Phys. Lett. B 243 (1990) 378 [InSPIRE].

[36] E. Sezgin and P. Sundell, Analysis of higher spin field equations in four-dimensions, JHEP 07 (2002) 055 [hep-th/0205132] [INSPIRE].

[37] N.S. Craigie, V.K. Dobrev and I.T. Todorov, Conformally covariant composite operators in quantum chromodynamics, Annals Phys. 159 (1985) 411 [INSPIRE].

[38] O.A. Gelfond, E.D. Skvortsov and M.A. Vasiliev, Higher spin conformal currents in Minkowski space, Theor. Math. Phys. 154 (2008) 294 [Teor. Mat. Fiz. 154 (2008) 344] [hep-th/0601106] [INSPIRE].

[39] O.A. Gelfond and M.A. Vasiliev, Operator algebra of free conformal currents via twistors, Nucl. Phys. B 876 (2013) 871 [arXiv:1301.3123] [inSPIRE].

[40] V.E. Didenko, J. Mei and E.D. Skvortsov, Exact higher-spin symmetry in CFT: free fermion correlators from Vasiliev theory, Phys. Rev. D 88 (2013) 046011 [arXiv:1301.4166] [INSPIRE].

[41] A.N. Vasiliev, M. Pismak, Yu and Yu. R. Khonkonen, Simple method of calculating the critical indices in the 1/N expansion, Theor. Math. Phys. 46 (1981) 104 [Teor. Mat. Fiz. 46 (1981) 157] [INSPIRE].

[42] S.E. Derkachov and A.N. Manashov, The simple scheme for the calculation of the anomalous dimensions of composite operators in the $1 / N$ expansion, Nucl. Phys. B 522 (1998) 301 [hep-th/9710015] [INSPIRE].

[43] T. Muta and D.S. Popovic, Anomalous dimensions of composite operators in the Gross-Neveu model in two $+\epsilon$ dimensions, Prog. Theor. Phys. 57 (1977) 1705 [InSPIRE].

[44] A.N. Manashov and E.D. Skvortsov, Higher-spin currents in the Gross-Neveu model at $1 / n^{2}$, JHEP 01 (2017) 132 [arXiv:1610.06938] [INSPIRE].

[45] V.E. Didenko and E.D. Skvortsov, Elements of Vasiliev theory, arXiv:1401.2975 [INSPIRE].

[46] M.A. Vasiliev, Consistent equations for interacting massless fields of all spins in the first order in curvatures, Annals Phys. 190 (1989) 59 [INSPIRE].

[47] M.A. Vasiliev, Extended higher spin superalgebras and their realizations in terms of quantum operators, Fortsch. Phys. 36 (1988) 33 [INSPIRE].

[48] S.E. Konstein and M.A. Vasiliev, Extended higher spin superalgebras and their massless representations, Nucl. Phys. B 331 (1990) 475 [INSPIRE].

[49] R.R. Metsaev, Cubic interaction vertices of massive and massless higher spin fields, Nucl. Phys. B 759 (2006) 147 [hep-th/0512342] [INSPIRE]. 
[50] E. D'Hoker and D.Z. Freedman, Supersymmetric gauge theories and the AdS/CFT correspondence, in Strings, Branes and Extra Dimensions: TASI 2001: Proceedings, Boulder CO U.S.A., 3-29 June 2001, pg. 3 [hep-th/0201253] [INSPIRE].

[51] B. Allen and T. Jacobson, Vector two point functions in maximally symmetric spaces, Commun. Math. Phys. 103 (1986) 669 [INSPIRE].

[52] D.Z. Freedman, S.D. Mathur, A. Matusis and L. Rastelli, Correlation functions in the $C F T_{d} / A d S_{d+1}$ correspondence, Nucl. Phys. B 546 (1999) 96 [hep-th/9804058] [INSPIRE].

[53] N. Boulanger, E. Sezgin and P. Sundell, $4 D$ higher spin gravity with dynamical two-form as a Frobenius-Chern-Simons gauge theory, arXiv:1505.04957 [INSPIRE].

[54] S.V. Mikhailov and A.V. Radyushkin, Evolution kernel for the pion wave function: two loop calculation in scalar $\phi^{3}$ in six-dimensions model, Theor. Math. Phys. 65 (1986) 999 [Teor. Mat. Fiz. 65 (1985) 44] [INSPIRE].

[55] C. Sleight and M. Taronna, Higher-spin algebras, holography and flat space, JHEP 02 (2017) 095 [arXiv: 1609.00991] [INSPIRE].

[56] S. Giombi and X. Yin, On higher spin gauge theory and the critical $O(N)$ model, Phys. Rev. D 85 (2012) 086005 [arXiv:1105.4011] [inSPIRE].

[57] X. Bekaert, E. Joung and J. Mourad, Comments on higher-spin holography, Fortsch. Phys. 60 (2012) 882 [arXiv:1202.0543] [INSPIRE].

[58] E.D. Skvortsov, On (un)broken higher-spin symmetry in vector models, in Proceedings, International Workshop on Higher Spin Gauge Theories, Singapore, 4-6 November 2015, pg. 103 [arXiv: 1512.05994] [INSPIRE].

[59] C. Sleight and M. Taronna, Higher spin interactions from conformal field theory: the complete cubic couplings, Phys. Rev. Lett. 116 (2016) 181602 [arXiv:1603.00022] [InSPIRE].

[60] X. Bekaert, J. Erdmenger, D. Ponomarev and C. Sleight, Quartic AdS interactions in higher-spin gravity from conformal field theory, JHEP 11 (2015) 149 [arXiv:1508.04292] [INSPIRE].

[61] C. Sleight and M. Taronna, Higher spin gauge theories and bulk locality: a no-go result, arXiv: 1704.07859 [INSPIRE].

[62] C. Sleight and M. Taronna, Spinning Witten diagrams, JHEP 06 (2017) 100 [arXiv: 1702.08619] [INSPIRE].

[63] G. Barnich and M. Henneaux, Consistent couplings between fields with a gauge freedom and deformations of the master equation, Phys. Lett. B 311 (1993) 123 [hep-th/9304057] [INSPIRE].

[64] S.F. Prokushkin and M.A. Vasiliev, Cohomology of arbitrary spin currents in AdS $S_{3}$, Theor. Math. Phys. 123 (2000) 415 [Teor. Mat. Fiz. 123 (2000) 3] [hep-th/9907020] [INSPIRE].

[65] P. Kessel, G. Lucena Gómez, E. Skvortsov and M. Taronna, Higher spins and matter interacting in dimension three, JHEP 11 (2015) 104 [arXiv: 1505.05887] [INSPIRE].

[66] M. Taronna, A note on field redefinitions and higher-spin equations, J. Phys. A 50 (2017) 075401 [arXiv: 1607.04718] [INSPIRE]. 
[67] A.A. Sharapov and E.D. Skvortsov, Formal higher-spin theories and Kontsevich-Shoikhet-Tsygan formality, Nucl. Phys. B 921 (2017) 538 [arXiv:1702.08218] [INSPIRE].

[68] M. Taronna, Higher-spin theories and locality, talk given at Higher-spin theory and holography, 6, http://www.hsth.lpi.ru/proceedings/taronna.pdf, Lebedev Institute, Moscow Russia, 31 May-2 June 2017.

[69] V.E. Didenko and M.A. Vasiliev, Test of the local form of higher-spin equations via $A d S / C F T$, arXiv:1705.03440 [INSPIRE]. 